Chronic Obstructive Pulmonary Diseases: Journal of the COPD Foundation

\author{
Original Research
}

\title{
Relationship Between FEV 1 and Patient-Reported Outcomes Changes: Results of a Meta-Analysis of Randomized Trials in Stable COPD
}

\author{
Christine de la Loge, $\mathrm{MSc}^{1}$ Béatrice Tugaut, $\mathrm{MSc}^{1}$ Fatoumata Fofana, $\mathrm{MSc}^{1}$ Jérémy Lambert, $\mathrm{PhD}^{1}$ \\ Michael Hennig, $\mathrm{PhD}^{2}$ Uta Tschiesner, $\mathrm{MD}^{3}$ Mitra Vahdati-Bolouri, MRCP, MFPM ${ }^{4}$ Afisi Segun Ismaila, $\mathrm{PhD}^{5}$ \\ Yogesh Suresh Punekar, $\mathrm{PhD}^{6}$
}

\begin{abstract}
Background: This meta-analysis assessed the relationship between change from baseline (CFB) in spirometric measurements (trough forced expiratory volume in 1 second [FEV 1 and $F E V_{1}$ area under the curve [AUC]) and patient-reported outcomes (St. George's Respiratory Questionnaire total score [SGRQ] CFB, Transition Dyspnea Index [TDI] and exacerbation rates) after 6-12 months' follow-up, using study treatment-group level data.

Methods: A systematic literature search was performed for randomized controlled trials of $\geq 24$ weeks duration in adults with chronic obstructive pulmonary disease (COPD). Studies reporting $\geq 1$ spirometric measurement and $\geq 1$ patient-reported outcome (PRO) at baseline and at study endpoint were selected. The relationships between PROs and spirometric endpoints were assessed using Pearson correlation coefficient and meta-regression.

Results: Fifty-two studies (62,385 patients) were included. Primary weighted analysis conducted at the last assessment showed a large significant negative correlation ( $\mathrm{r},-0.68$ [95\% confidence interval (CI); -0.77, -0.57]) between trough $\mathrm{FEV}_{1}$ and $S \mathrm{GRQ}$. Improvement of $100 \mathrm{~mL}$ in trough $\mathrm{FEV}$ corresponded to a 5.9 point reduction in SGRQ. Similarly, a reduction of 4 points on SGRQ corresponded to $40 \mathrm{~mL}$ improvement in trough FEV $\mathrm{F}_{1}(p<0.001)$. The weighted correlation coefficients of trough FEV 1 with TDI, exacerbation rate (all) and exacerbation rate (moderate/severe) at last assessment point were $0.57,-0.69$ and -0.57 , respectively (all $p<0.05$ ). For the analyses excluding placebo groups, the correlations of FEV 1 with SGRQ and TDI were lower but significant.

Conclusions: A strong association exists between changes in spirometric measurements and changes in PROs.
\end{abstract}

Abbreviations: change from baseline, CFB; forced expiratory volume in 1 second, FEV $\mathbf{1}$; area under the curve, AUC; St. George's Respiratory Questionnaire total score, SGRQ; transition dyspnea index, TDI; chronic obstructive pulmonary disease, COPD; patient-reported outcome, PRO; confidence interval, CI; Global initiative for chronic Obstructive Lung Disease, GOLD; randomized controlled trial, RCT; long-acting muscarinic antagonist, LAMA; long-acting $\beta 2$-agonist, LABA; minimal clinically important difference, MCID; Cochrane Database of Systematic Review, CDSR; Cochrane Central Register of Controlled Trials, CENTRAL; Database of Abstracts of Reviews of Effects, DARE; EU Clinical Trials Register, EU-CTR; health technology assessment, HTA; National Institute for Health Research-Health Technology Assessment, NIHR-HTA; World Health Organization International Clinical Trials Registry Platform, WHO ICTRP; standard deviation, SD; baseline dyspnea index, BDI; 2 times a day, BID; not applicable, NA; once a day, OD; 4 times a day, QID

Funding Support: This study (GK15920) was funded by GlaxoSmithKline.

Date of Acceptance: December 9, 2015

Citation: de la Loge C, Tugaut B, Fofana F, et al. Relationship between FEV 1 and patient-reported outcomes changes: Results of a metaanalysis of randomized trials in stable COPD. Chronic Obstr Pulm Dis (Miami). 2016; 3(2):519-538. doi: http://dx.doi.org/10.15326/ jcopdf.3.2.2015.0152

\section{This article has an online data supplement.}




\begin{abstract}
1 Mapi, Patient-Centered Outcomes, Lyon, France
2 Biostatistics and Epidemiology, GlaxoSmithKline, Munich, Germany

3 Former employee of GlaxoSmithKline, Munich, Germany

4 Research and Development, Global Respiratory Franchise, GlaxoSmithKline, Brentford, United Kingdom

5 Value Evidence and Outcomes, GlaxoSmithKline Research and Development, Research Triangle Park, North Carolina; Clinical Epidemiology and Biostatistics, McMaster University, Hamilton, Ontario, Canada

6 Value Evidence and Outcomes, GlaxoSmithKline, Brentford, United Kingdom
\end{abstract}

\section{Address correspondence to:}

Yogesh Suresh Punekar, PhD

GlaxoSmithKline

Brentford, TW8 9GS, UK

Email: yogesh.q.punekar@gsk.com

Phone: +44 (0) 2080474264

\section{Keywords:}

$\mathrm{FEV}_{1}$; SGRQ; TDI; exacerbation; COPD; systematic literature review; meta-analysis; correlation; linear regression; patientreported outcomes

\section{Introduction}

Chronic obstructive pulmonary disease (COPD) is a progressive disease of the respiratory system characterized by chronic airway inflammation. The resulting airflow limitation is not fully reversible. Disease progression is associated with more severe and frequent exacerbations and declining lung function. ${ }^{1}$ Nevertheless, COPD is frequently under-diagnosed and under-treated.

The global burden of COPD is high and by 2020 will increase to reach a rank of 5 for burden of disease and 3 for cause of death. ${ }^{2}$ According to Global initiative for chronic Obstructive Lung Disease (GOLD) recommendations, assessment of COPD is based on the patient's level of symptoms, exacerbation history, severity of spirometric abnormality, and identification of comorbidities. ${ }^{2}$ Although spirometry is now required for a confident diagnosis of COPD, diagnosis and management of the disease should not be purely based on spirometric categorization. Given the evidence that the level of forced expiratory volume in 1 second $\left(F E V_{1}\right)$ poorly represents COPD status, revised GOLD guidelines recommend that both disease impact (symptom burden and activity limitation) and future risk of disease progression, particularly exacerbations, must be considered for adequate management of stable

\section{COPD. $^{2}$}

Patient-reported outcomes ( $\mathrm{PROs}$ ) based on symptom severity, activity limitation or health status are highly relevant for assessing disease severity or treatment impact from the perspective of policy makers and payers. $^{3,4}$ Such outcomes are routinely collected in clinical trials using fully validated and widely accepted PRO instruments such as St. George's Respiratory Questionnaire (SGRQ) and the Transition Dyspnea Index (TDI). However, there is limited evidence on the relationship between the typical regulatory endpoints such as FEV 1 and the PRO endpoints which often creates challenges for policy makers while making reimbursement decisions for specific treatments.

The primary objective of the study was to assess the relationship between changes in spirometric measurements (particularly trough $\mathrm{FEV}_{1}$ ) and changes in PROs (SGRQ, TDI, and exacerbation rates) after at least 6 months of follow-up, using study treatment group level data. The analysis was repeated using treatment arms with active treatments (excluding placebo groups) and using treatment effect measurements (difference over placebo) for placebo-controlled studies.

\section{Methods}

\section{Search Strategy}

A systematic literature review was performed using a predefined search strategy to identify randomized controlled trials (RCTs) of 24 weeks' duration or more in patients with COPD. Independent bibliographic systematic searches were conducted in April 2014 using the following databases (from inception to April 2014): MEDLINE, MEDLINE In-Process, EMBASE, the Cochrane Library, Database of Abstracts of Reviews of Effects, and Health Technology Assessment websites. Secondary systematic searches in clinical trial registries such as Clinicaltrials.gov (the U.S. National Institutes of Health clinical trial register), World Health Organization International Clinical Trials Registry Platform, International Standard Randomised Controlled Trial Number registry, and the European and Clinical Trials Register, were performed. Experienced researchers developed search strategies specifically tailored for each database. As an example, the search strategy for MEDLINE and MEDLINE In-Process is provided in Appendix 1 of the online supplementary data.

\section{Selection Criteria}

RCTs of at least 24 weeks' duration conducted in adults 
with COPD (per GOLD guidelines) receiving long-acting muscarinic antagonists (LAMAs) and/or long-acting $\beta 2$-agonists (LABAs) were included. Furthermore, only studies reporting at least 1 spirometric measurement of interest (trough $\mathrm{FEV}_{1}$, time-adjusted $\mathrm{FEV}_{1} \mathrm{AUC}$ ) and at least one PRO of interest (SGRQ, TDI, and exacerbation rates) at baseline and 6 and/or 12 months were selected. The search was limited to English language.

The search was directed to studies with similar pharmacodynamics properties: studies of monotherapy with LAMAs or LABAs (monotherapy with aclidinium bromide, formoterol, glycopyrronium, indacaterol, salmeterol, tiotropium, umeclidinium, or vilanterol) and/or the fixed-dose or free combination of both (umeclidinium/vilanterol, aclidinium/formoterol, tiotropium/olodaterol, or indacaterol/glycopyrronium). Studies with any of these treatments were included.

Studies were excluded if: (a) data were not available simultaneously for spirometric measurement and PRO endpoints at any time-point of interest; (b) the reported $\mathrm{FEV}_{1}$ was measured postdose; or (c) there was no evidence that $\mathrm{FEV}_{1}$ was measured predose. Furthermore, studies limited to patients with alpha-1 antitrypsin deficiency-related COPD and to non-white populations (e.g., Chinese, Japanese patients) were excluded.

The SGRQ assesses 3 domains (symptoms, activity, and impacts), with a total score ranging between 0 and 100. Higher values of SGRQ are associated with lower health-related quality of life.,6 TDI characterizes a change in dyspnea from baseline and provides values between -9 and $9{ }^{7}$ Positive values in the TDI score correspond to clinical improvement. A 4-unit change in the total score of the SGRQ, ${ }^{8}$ a 1 -unit change in TDI, ${ }^{9}$ and a change of $100 \mathrm{~mL}$ in $\mathrm{FEV}_{1}{ }^{10}$ are considered as minimal clinically important differences (MCIDs) for these instruments. There is no agreed MCID for exacerbation rates although several estimates have been reported in literature. ${ }^{11}$

\section{Selection Process}

The relevance of each identified citation was assessed according to the predefined selection criteria. Selection was performed by 2 researchers (BT and $\mathrm{JL}$ ) independently along with standardized quality assessments of the selected studies. Any discrepancies between researchers were resolved by consensus. The selected citations were grouped per study, as 1 study could have been published in several sources such as a conference abstract, full-text article, or trial registration.

\section{Data Extraction}

Data extraction was performed by 2 researchers (BT and JL) independently. Any discrepancies were discussed and resolved by consensus.

Data were primarily extracted from the text and tables of the source documents. If the data of interest were available solely as figures, these were extracted using DigitizeIt software version 2.0.3 (Digitize It, Braunschweig, Germany, http://digitizeit.de ). For each study, study characteristics, population characteristics, treatment groups, and spirometric and PRO endpoints of interest at selected time points (mean CFB, mean baseline, and mean follow-up values) were extracted. If mean CFB values were unavailable, these were calculated by subtracting the mean value at baseline from the mean value at follow-up.

\section{Statistical Analysis}

Study and patient characteristics, as well as outcome results (spirometric measurements and PROs at 6 or 12 months follow-up and last assessment) were summarized across all studies using (1) weights proportional to the sample size of the study treatment group in relation to the total number of patients across all treatment groups (weighted approach), and (2) equal weights for each study treatment group (unweighted approach).

Methods used to assess the relationship between $\mathrm{PROs}$ and spirometric endpoints included scatter and bubble plots ( 1 dot representing a treatment group results for both endpoints considered; the size of the dot being proportional to the sample size of the considered treatment group), linear regressions, and Pearson correlation coefficients with 95\% confidence interval (CI). The linear regression equations were used to estimate the mean change in $\mathrm{FEV}_{1}$ corresponding to the established MCID thresholds of the PROs and to estimate the mean change in PROs corresponding to the established MCID threshold of a 100-mL change in $\mathrm{FEV}_{1}{ }^{10}$ Similarly, the rate and incidence of exacerbations corresponding to a change of $100 \mathrm{~mL}$ in $\mathrm{FEV}_{1}$ also were calculated.

Primary analysis involved quantifying the relationship between trough FEV 1 CFB and SGRQ CFB at last assessment (i.e., assessment at the 12-month followup if available for both considered endpoints, or if not available, at the 6-month follow-up).

Further statistical analyses were conducted to facilitate interpretation of results and explore the data. The regression and correlation analyses were conducted 
after exclusion of the placebo groups. We also conducted regression and correlation analyses between the active treatment group effect beyond placebo in $\mathrm{FEV}_{1} \mathrm{CFB}$ and the effect beyond placebo in the various PROs (analyses conducted using data from placebo-controlled studies only, where the placebo group result is subtracted from each treatment group result).

All these analyses were conducted only when data for at least 15 study treatment groups were available. Such a sample size allows detecting a correlation coefficient of 0.7 with more than $85 \%$ power and associated type I error of $0.05 .^{12}$ Interpretation of the amplitude of the absolute values of correlation coefficients were based on Cohen's conventions (0.1-0.3, small/ weak; 0.3-0.5, medium/moderate; >0.5, large). ${ }^{12}$ No statistical correction for multiple tests was performed. All statistical analyses were conducted based on a predefined statistical analysis plan and using SAS software for Windows (Version 9.2, SAS Institute, Inc., Cary, NC, USA).

\section{Results}

\section{Literature Search}

The systematic bibliographic search identified 3006 abstracts from which a total of 2515 were excluded in the abstract/title screening phase. After full-text screening, a further 261 publications were excluded. The systematic registry search identified 4720 trial registrations from which 4636 were excluded (Figure 1). Three additional recently published references were identified through conference abstract and the registry search. Therefore, 233 full text publications and 84 trial registrations were retained for final study selection.

Overall, 118 studies were identified from the citations extracted based on the systematic literature search. Thirty-nine studies from the registry search did not have any results published or posted on the registry websites at the time of the search. The outcomes of 27 studies were out of scope of present meta-analysis; these studies were also excluded. In total, 52 unique studies ${ }^{13-62}$ were selected for this meta-analysis and the data for all these studies were extracted from all available sources, including clinical trial registries.

\section{Study Characteristics}

A description of key study characteristics is summarized in Table 1. The 52 unique studies included 163 treatment groups and 62,385 patients. The median study duration was 11.7 months. A majority of the studies (80.8\%) did not allow background LABA and $57.7 \%$ allowed background ICS treatment. A majority of studies considered a lower threshold inclusion criterion of 10 pack years of cigarette smoking (82.7\%) but no inclusion criteria regarding the number of exacerbations over the past year (71.2\%). The upper thresholds most commonly encountered for the percentage of $\mathrm{FEV}_{1}$ inclusion criterion were $80 \%$ (28.8\%) and $70 \%$ (23.1\%).

\section{Population Baseline Characteristics}

The patients' characteristics weighted by the sample size of each group across the 163 treatment groups from the 52 selected studies are summarized in Table 2 . The number of patients in each study treatment group varied from 6 to 3006, with a median of 419. The mean (standard deviation [SD]) age was 63.7 (25.0) years. The proportion of men across the treatment groups varied from $43.0 \%$ to $100.0 \%$ (weighted mean proportion $70.4 \%$ ). Large variation in baseline characteristics was seen for disease severity with the percentage of patients classified as severe or very severe (GOLD stage III or IV) ranging from $19.7 \%$ to $100.0 \%$ (median, $53.0 \%$ ) and mean baseline trough $\mathrm{FEV}_{1}$ ranging from 890 to $1681 \mathrm{~mL}$ (median, $1180 \mathrm{~mL}$ ).

Most treatment groups were receiving LABA (25.2\%), LAMA (21.5\%), placebo (20.9\%), or LABA and ICS (19.6\%).

\section{Data Availability for the Endpoint Combinations}

The online supplementary Table 1 provides treatment group-level data on endpoints of interest for all the included studies. The combinations of endpoints with at least 15 study-treatment groups $(\mathrm{N})$ are described in Table 3. In combination with $\mathrm{FEV}_{1}$, SGRQ was the most reported endpoint (111 treatment groups; 38 studies) followed by TDI $(68 ; 22)$, all exacerbations $(24 ; 10)$ and moderate/severe exacerbations (69; 23). FEV 1 AUC $0-12 \mathrm{~h}$ and SGRQ data at last assessment were available from 5 studies with 22 treatment arms.

The duration between baseline and the last assessment varied across endpoint combinations. The duration was longest for the analysis of the combination of SGRQ with trough $\mathrm{FEV}_{1}$ (median, 11.1 months; 55.9\% at 12 months) and shortest for the analysis of $\mathrm{FEV}_{1}$ AUC $_{0-12 \mathrm{~h}}$ with trough $\mathrm{FEV}_{1}$ (median, 6.0 months; $81.8 \%$ at 6 months).

\section{Correlation and Regression Analyses Between Spirometric Measurements and PROs}

The correlation and regression results of the primary 


\section{Figure 1. Flow of Studies Through the Review Process}

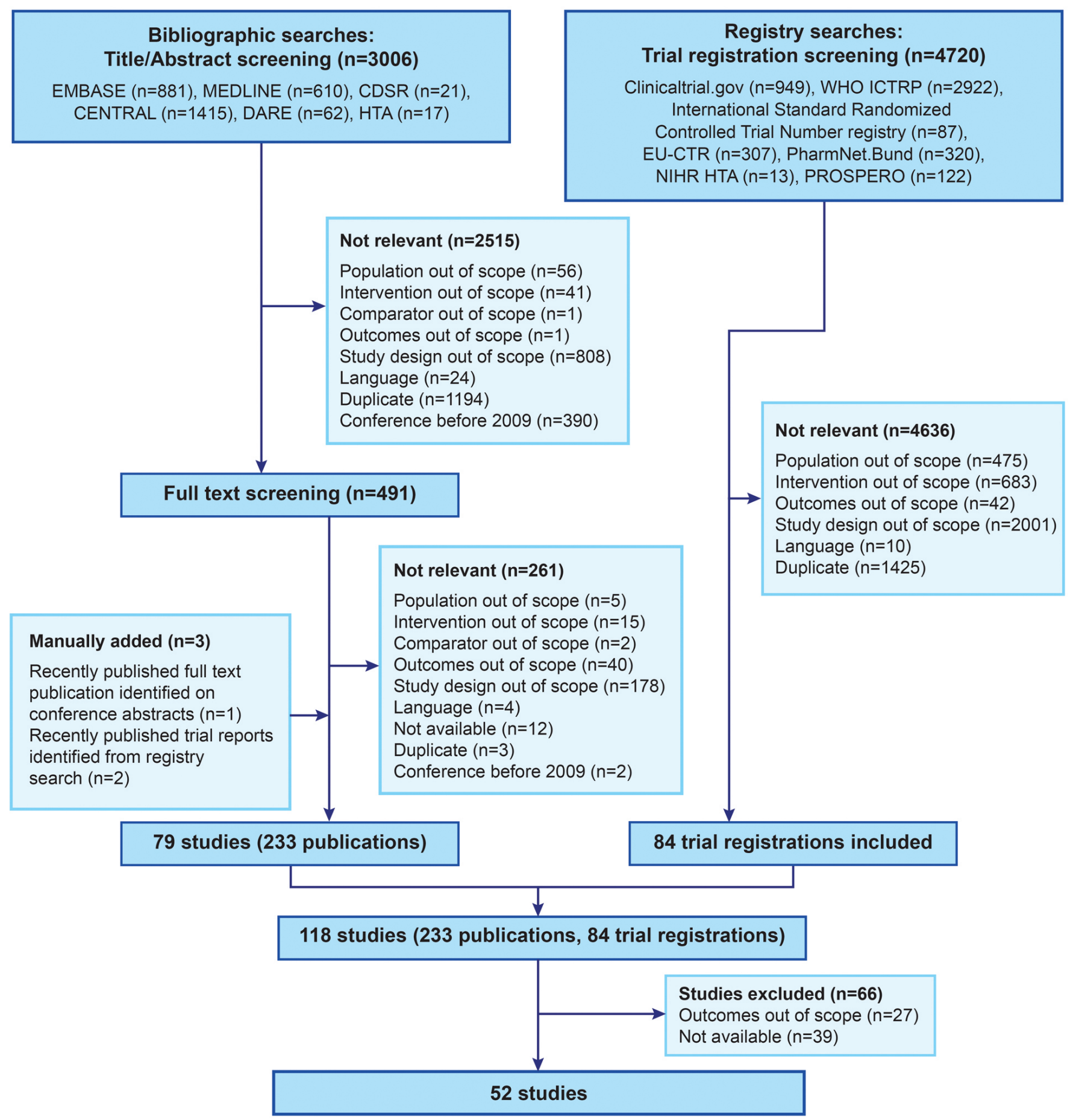

CDSR=Cochrane Database of Systematic Review; CENTRAL $=$ Cochrane Central Register of Controlled Trials; DARE=Database of Abstracts of Reviews of Effects; EU-CTR=EU Clinical Trials Register; HTA=health technology assessment; NIHR-HTA=National Institute for Health Research-Health Technology Assessment; WHO ICTRP=World Health Organization International Clinical Trials Registry Platform 


\section{Table 1. Description of Key Baseline Characteristics at Study Level $^{\mathrm{a}}$}

\begin{tabular}{|c|c|c|c|c|c|c|c|c|c|}
\hline Study Name & Duration, ${ }^{\mathrm{b}}$ & $\begin{array}{l}\text { bumber } \\
\text { of } \\
\text { Treatment } \\
\text { Groups }\end{array}$ & $\begin{array}{c}\text { Total } \\
\text { Number of } \\
\text { Patients in } \\
\text { the Study }\end{array}$ & $\begin{array}{cc} & \text { Age } \\
f & \text { (years) } \\
\text { nean } \\
\text { Mean }\end{array}$ & $\begin{array}{l}\text { Men } \\
(\%)\end{array}$ & $\begin{array}{c}\text { Proportion } \\
\text { (\%) of Severe/ } \\
\text { Very Severe } \\
\text { COPD } \\
\text { Patients } \\
\text { Mean (SD) }\end{array}$ & $\begin{array}{l}\text { Trough } \\
\text { FEV }_{1} \\
\text { at Baseline } \\
(\mathrm{mL}) \\
\text { Mean (SD) }\end{array}$ & $\begin{array}{l}\text { BDI } \\
\text { Mean } \\
\text { (SD) }\end{array}$ & $\begin{array}{c}\text { SGRQ } \\
\text { Total } \\
\text { Score } \\
\text { Baseline } \\
\text { Mean (SD) }\end{array}$ \\
\hline $\begin{array}{l}\text { Celli et al, } \\
2014^{13}\end{array}$ & 24 & 4 & 1493 & $62.9(9.2)$ & $\begin{array}{c}65.4 \\
(20.4)\end{array}$ & $53.0(65.8)$ & & & \\
\hline $\begin{array}{l}\text { Abrahams et al, } \\
2013^{14}\end{array}$ & 24 & 5 & 2080 & $64.2(6.5)$ & $\begin{array}{c}64.5 \\
(17.6)\end{array}$ & & $1186.0(184.0)$ & $6.5(1.7)$ & $43.1(14.4)$ \\
\hline $\begin{array}{l}\text { Bateman et al, } \\
2013 \text { SHINE } 15\end{array}$ & 26 & 5 & 2144 & $63.91(8.1)$ & $\begin{array}{c}75.4 \\
(32.2)\end{array}$ & $36.3(49.9)$ & $1300.0(0.0)$ & $6.4(2.6)$ & $46.9(12.6)$ \\
\hline $\begin{array}{l}\text { Decramer et al, } \\
2013 \text { INVIGORATE } 16\end{array}$ & 52 & 2 & 3439 & & $\begin{array}{c}77.0 \\
(58.6)\end{array}$ & $99.0(0.0)$ & & $6.0(2.9)$ & $48.3(23.5)$ \\
\hline $\begin{array}{l}\text { Donohue et al, } \\
2013^{17}\end{array}$ & 24 & 4 & 1536 & $63.1(14.5)$ & $\begin{array}{c}70.8 \\
(50.8)\end{array}$ & $53.7(47.8)$ & & & \\
\hline $\begin{array}{l}\text { Dransfield et al, } \\
2013 \text { Study } 1^{18}\end{array}$ & 52 & 4 & 1622 & $63.7(2.0)$ & $\begin{array}{c}59.4 \\
(40.2)\end{array}$ & & $1220.0(257.1)$ & & \\
\hline $\begin{array}{l}\text { Dransfield et al, } \\
2013 \text { Study } 2^{18}\end{array}$ & 52 & 4 & 1633 & $63.7(4.4)$ & $\begin{array}{c}55.5 \\
(35.7)\end{array}$ & & $1235.9(387.3)$ & & \\
\hline $\begin{array}{l}\text { D'Urzo et al, } \\
2013^{19}\end{array}$ & 64 & 4 & 291 & $63.9(10.0)$ & $\begin{array}{c}51.9 \\
(23.4)\end{array}$ & & $1340.1(712.7)$ & & $44.3(27.9)$ \\
\hline $\begin{array}{l}\text { Gelb et al, } \\
2013^{20}\end{array}$ & 52 & 2 & 605 & $63.6(14.8)$ & $\begin{array}{c}58.3 \\
(22.1)\end{array}$ & & & & 49.1 (16.0) \\
\hline $\begin{array}{l}\text { Kerwin et al, } \\
2013^{21}\end{array}$ & 24 & 5 & 1030 & $62.7(7.2)$ & $\begin{array}{c}66.6 \\
(24.0)\end{array}$ & & $1238.4(641.6)$ & & \\
\hline $\begin{array}{l}\text { Martinez et al, } \\
2013^{22}\end{array}$ & 24 & 6 & 1224 & $61.6(5.2)$ & $\begin{array}{c}72.3 \\
(41.1)\end{array}$ & & $1353.8(524.9)$ & & \\
\hline $\begin{array}{l}\text { Vogelmeier et al, } \\
2013 \text { ILLUMINATE } 23\end{array}$ & 26 & 2 & 523 & $63.3(2.3)$ & $\begin{array}{c}70.9 \\
(16.0)\end{array}$ & $19.8(1.1)$ & 1449.5 (1143.4) & & \\
\hline $\begin{array}{l}\text { Wedzicha et al, } \\
2013 \text { SPARK }^{24}\end{array}$ & 64 & 3 & 2224 & $63.3(7.9)$ & $\begin{array}{c}74.7 \\
(41.6) \\
\end{array}$ & $100.0(0.0)$ & $900.0(272.3)$ & & $52.3(15.7)$ \\
\hline $\begin{array}{l}\text { Doherty et al, } \\
2012^{25}\end{array}$ & 52 & 5 & 1196 & 59.7 (10.6) & $\begin{array}{c}75.2 \\
(28.1)\end{array}$ & & $1204.1(485.8)$ & & $47.3(14.2)$ \\
\hline $\begin{array}{l}\text { Hanania et al, } \\
2012^{26}\end{array}$ & 24 & 2 & 342 & $61.2(2.8)$ & $\begin{array}{r}46.5 \\
(64.7)\end{array}$ & $32.6(83.2)$ & & & \\
\hline $\begin{array}{l}\text { Jones et al, } \\
2012 \text { ATTAIN }^{27}\end{array}$ & 24 & 3 & 828 & $62.4(7.6)$ & $\begin{array}{c}67.4 \\
(32.8) \\
\end{array}$ & $31.9(32.1)$ & & $6.8(2.9)$ & $46.3(20.7)$ \\
\hline $\begin{array}{l}\text { Kerwin et al, } \\
2012 \text { GLOW } 2^{28}\end{array}$ & 52 & 3 & 1066 & $63.6(3.8)$ & $\begin{array}{c}64.2 \\
(17.0)\end{array}$ & 35.9 (18.5) & $1325.2(1002.8)$ & & \\
\hline $\begin{array}{l}\text { Sharafkhaneh et al, } \\
2012^{29}\end{array}$ & $\begin{array}{c}12 \\
\text { months }\end{array}$ & 3 & 1219 & $63.0(13.7)$ & $\begin{array}{c}62.0 \\
(90.1)\end{array}$ & & 993.4 (419.2) & & 57.4 (27.9) \\
\hline $\begin{array}{l}\text { Tashkin et al, } \\
2012^{30}\end{array}$ & 52 & 5 & 1055 & $59.8(10.9)$ & $\begin{array}{c}77.6 \\
(39.2)\end{array}$ & & $1230.5(389.1)$ & & $45.5(14.2)$ \\
\hline $\begin{array}{l}\text { Chapman et al, } \\
2011^{31}\end{array}$ & 52 & 3 & 415 & $62.6(2.0)$ & $\begin{array}{c}61.2 \\
(36.9)\end{array}$ & & & & $45.5(6.0)$ \\
\hline
\end{tabular}




\begin{tabular}{|c|c|c|c|c|c|c|c|c|c|}
\hline $\begin{array}{l}\text { D'Urzo et al, } \\
2011 \text { GLOW } 1^{32}\end{array}$ & 26 & 2 & 822 & $63.9(2.7)$ & $\begin{array}{l}81.8 \\
(26.9)\end{array}$ & $39.2(28.3)$ & $1320.3(807.9)$ & & $46.2(3.1)$ \\
\hline $\begin{array}{l}\text { Jones et al, } 2011 \\
\text { ACCLAIM/COPD I33 }\end{array}$ & 52 & 2 & 843 & $62.4(8.9)$ & $\begin{array}{l}78.6 \\
(40.6)\end{array}$ & & $1405.8(354.9)$ & $6.3(1.3)$ & $47.3(0.0)$ \\
\hline $\begin{array}{l}\text { Jones et al, } 2011 \\
\text { ACCLAIM/COPD II }{ }^{33}\end{array}$ & 52 & 2 & 804 & $65.1(1.2)$ & $\begin{array}{c}63.0 \\
(37.0)\end{array}$ & & $1198.8(740.3)$ & $6.4(3.7)$ & $45.7(23.4)$ \\
\hline $\begin{array}{l}\text { Bateman et al, } \\
2010 \mathrm{a}^{34}\end{array}$ & 48 & 3 & 1990 & $65.0(6.8)$ & $\begin{array}{l}74.2 \\
(20.2)\end{array}$ & & & & \\
\hline $\begin{array}{l}\text { Bateman et al, } \\
2010 b^{35}\end{array}$ & 48 & 2 & 3917 & $64.8(0.0)$ & $\begin{array}{l}77.6 \\
(34.4)\end{array}$ & & $1105.0(250.3)$ & & \\
\hline $\begin{array}{l}\text { Calverley et al, } \\
2010^{36}\end{array}$ & 48 & 3 & 718 & $63.6(8.6)$ & $\begin{array}{c}80.6 \\
(18.1)\end{array}$ & $100.0(0.0)$ & $1146.7(179.1)$ & & $50.3(9.7)$ \\
\hline $\begin{array}{l}\text { Dahl et al, } \\
2010 \text { INVOLVE } \\
27\end{array}$ & 52 & 4 & 1732 & 63.5 (12.0) & $\begin{array}{l}79.7 \\
(40.9)\end{array}$ & 45.7 (30.6) & $1287.6(199.2)$ & $6.0(0.0)$ & $43.5(12.0)$ \\
\hline $\begin{array}{l}\text { Donohue et al, } \\
2010 \text { INHANCE } 38\end{array}$ & 26 & 4 & 1683 & $63.6(6.3)$ & $\begin{array}{c}62.8 \\
(32.8)\end{array}$ & & $1305.0(1188.1)$ & & \\
\hline $\begin{array}{l}\text { Hanania et al, } \\
2010^{39}\end{array}$ & $\begin{array}{c}6 \\
\text { months }\end{array}$ & 3 & 443 & 64.4 (10.9) & $\begin{array}{c}61.2 \\
(52.9)\end{array}$ & & $1212.1(111.6)$ & $5.3(1.7)$ & $50.2(23.2)$ \\
\hline $\begin{array}{l}\text { Anzueto et al, } \\
2009^{40}\end{array}$ & 52 & 2 & 797 & $65.4(1.4)$ & $\begin{array}{c}54.0 \\
(84.7)\end{array}$ & & 975.1 (141.2) & & \\
\hline $\begin{array}{l}\text { Rennard et al, } \\
2009^{41}\end{array}$ & $\begin{array}{c}12 \\
\text { months }\end{array}$ & 4 & 1964 & $63.2(7.4)$ & $\begin{array}{c}63.9 \\
(35.5)\end{array}$ & $82.0(22.8)$ & $1024.5(1100.3)$ & & $55.0(11.1)$ \\
\hline $\begin{array}{l}\text { Donohue et al, } \\
2008^{42}\end{array}$ & 52 & 2 & 793 & $64.0(14.6)$ & $\begin{array}{c}58.9 \\
(51.8)\end{array}$ & & $1136.6(531.3)$ & & \\
\hline $\begin{array}{l}\text { Ferguson et al, } \\
2008^{43}\end{array}$ & $\begin{array}{c}12 \\
\text { months }\end{array}$ & 2 & 782 & $65.0(1.4)$ & $\begin{array}{l}55.0 \\
(83.9)\end{array}$ & $100.0(0)$. & 940.1 (279.6) & & \\
\hline $\begin{array}{l}\text { Tashkin et al, } \\
2008^{44}\end{array}$ & 26 & 6 & 1704 & $63.4(3.9)$ & $\begin{array}{c}68.1 \\
(57.9)\end{array}$ & $80.1(20.0)$ & $1045.4(338.5)$ & & $55.7(17.8)$ \\
\hline $\begin{array}{l}\text { Tashkin et al, } \\
2008 \text { UPLIFT }^{45}\end{array}$ & $\begin{array}{c}4 \\
\text { years }\end{array}$ & 2 & 5993 & $64.5(0.0)$ & $\begin{array}{c}74.7 \\
(58.1)\end{array}$ & $52.5(38.7)$ & $1095.0(387.1)$ & & $45.9(11.6)$ \\
\hline $\begin{array}{l}\text { Tonnel et al, } \\
2008 \text { TIPHON } 46\end{array}$ & $\begin{array}{c}9 \\
\text { months }\end{array}$ & 2 & 554 & $64.2(16.5)$ & $\begin{array}{l}86.1 \\
(16.8)\end{array}$ & 59.5 (53.9) & $1364.4(352.8)$ & & 47.4 (36.5) \\
\hline $\begin{array}{l}\text { Aaron et al, } \\
2007^{47}\end{array}$ & 52 & 3 & 449 & $67.7(4.0)$ & $\begin{array}{c}56.3 \\
(27.6)\end{array}$ & & $1016.2(350.3)$ & & $49.1(19.1)$ \\
\hline $\begin{array}{l}\text { Chan et al, } \\
2007 \text { SAFE }^{48}\end{array}$ & 48 & 2 & 913 & $66.8(1.4)$ & $\begin{array}{l}59.7 \\
(28.5)\end{array}$ & & 966.7 (142.5) & & $46.4(0.0)$ \\
\hline $\begin{array}{l}\text { Stockley et al, } \\
2006^{49}\end{array}$ & 52 & 2 & 634 & $62.4(1.3)$ & $\begin{array}{l}76.5 \\
(12.6)\end{array}$ & & $1324.0(226.6)$ & & $49.1(18.9)$ \\
\hline $\mathrm{SCO} 100470^{50}$ & 24 & 2 & 1050 & $63.6(3.2)$ & $\begin{array}{l}77.8 \\
(18.2)\end{array}$ & & $1667.7(437.4)$ & $5.6(0.0)$ & 48.1 (3.2) \\
\hline SLMF $4010^{51}$ & 24 & 2 & 34 & $63.8(5.0)$ & $\begin{array}{l}88.2 \\
(34.3)\end{array}$ & & & & \\
\hline $\begin{array}{l}\text { Wouters et al, } \\
2005 \text { COSMIC }^{52}\end{array}$ & $\begin{array}{c}12 \\
\text { months }\end{array}$ & 2 & 373 & $63.5(9.7)$ & $\begin{array}{l}74.0 \\
(19.3)\end{array}$ & & $1410.0(0.0)$ & & $39.1(9.7)$ \\
\hline $\begin{array}{l}\text { Brusasco et al, } \\
2003^{53}\end{array}$ & 24 & 3 & 1207 & $64.2(8.1)$ & $\begin{array}{l}76.3 \\
(23.1)\end{array}$ & & $1093.3(505.4)$ & & \\
\hline $\begin{array}{l}\text { Calverley et al, } \\
2003 \text { TRISTAN54 }\end{array}$ & 52 & 4 & 1465 & $63.2(6.8)$ & $\begin{array}{c}72.5 \\
(55.2)\end{array}$ & & $1269.4(513.8)$ & & $48.2(25.3)$ \\
\hline
\end{tabular}




\begin{tabular}{|c|c|c|c|c|c|c|c|c|}
\hline $\begin{array}{l}\text { Dal Negro et al, } \\
2003^{55}\end{array}$ & 52 & 3 & 18 & & $\begin{array}{l}88.9 \\
(23.6)\end{array}$ & $1453.3(92.7)$ & & \\
\hline $\begin{array}{l}\text { Hanania et al, } \\
2003^{56}\end{array}$ & 24 & 4 & 723 & $63.8(13.0)$ & $\begin{array}{c}63.3 \\
(61.5)\end{array}$ & 1220.3 (218.6) & $6.0(3.0)$ & \\
\hline $\begin{array}{l}\text { Casaburi et al, } \\
2002^{57}\end{array}$ & 49 & 2 & 921 & $65.0(0.0)$ & $\begin{array}{c}65.0 \\
(55.7)\end{array}$ & 1023.9 (595.4) & $6.1(3.0)$ & \\
\hline $\begin{array}{l}\text { Donohue et al, } \\
2002^{59}\end{array}$ & 24 & 3 & 623 & $64.9(8.7)$ & $\begin{array}{l}74.7 \\
(8.3)\end{array}$ & 1080.2 (380.6) & $6.5(3.5)$ & $45.4(0.0)$ \\
\hline $\begin{array}{l}\text { Mahler et al, } \\
2002^{60}\end{array}$ & 24 & 4 & 674 & 63.5 (14.2) & $\begin{array}{c}65.7 \\
(85.8)\end{array}$ & 1226.9 (667.3) & $6.0(2.2)$ & \\
\hline
\end{tabular}

aBlank cells represent information that was not recorded.

${ }^{b}$ The duration of study is in weeks unless specified otherwise.

\section{Table 2. Key Baseline Characteristics Summarized Across All Study Treatment Groups (Weighted by Treatment Group Sample Size)}

\begin{tabular}{l|r|rrr}
\hline Characteristics & $\begin{array}{c}\text { Number } \\
\text { of Study } \\
\text { Treatment } \\
\text { Groups (\%) }\end{array}$ & Mean (SD) & Median & $\begin{array}{c}\text { Minimum, } \\
\text { Maximum }\end{array}$ \\
\hline Number of Patients per Treatment Group & & & \\
\hline Age (years) - mean & $163(100.0)$ & $809.9(16959.0)$ & 419.0 & 6,3006 \\
\hline Gender, Male (\%) & $158(96.9)$ & $63.7(25.0)$ & 63.7 & $58.8,68.1$ \\
\hline \% Current Smokers & $163(100.0)$ & $70.4(159.1)$ & 73.9 & $43.0,100.0$ \\
\hline Number of Pack Years of Cigarettes, mean & $133(81.6)$ & $40.8(153.8)$ & 40.0 & $0.0,59.0$ \\
\hline \% Patients with Moderate COPD & $114(69.9)$ & $46.1(103.7)$ & 45.7 & $33.2,63.0$ \\
\hline \% Patients with Severe or Very Severe COPD & $53(32.5)$ & $34.9(566.0)$ & 45.0 & $0.0,80.3$ \\
\hline Length of Time with COPD (years), mean & $53(32.5)$ & $64.4(573.5)$ & 53.0 & $19.7,100.0$ \\
\hline Baseline Trough FEV 1 (mL), mean & $70(42.9)$ & $8.7(34.6)$ & 8.5 & $5.9,11.4$ \\
\hline Baseline SGRQ Score, mean & $138(84.7)$ & $1177.0(2916.3)$ & 1180.0 & $890.0,1681.0$ \\
\hline Baseline Dyspnea Index, mean & $96(58.9)$ & $48.1(77.2)$ & 47.1 & $38.4,58.6$ \\
\hline
\end{tabular}

Note: Overall mean per study are weighted by the number of patients randomized in each treatment group within the study.

aThe total number of patients randomized in all studies is 62,385

and secondary analyses are shown in Tables 4-6 and Figures 2-4. Table 4 provides weighted and unweighted Pearson correlation coefficients and linear regression results showing values corresponding to known MCIDs for each combination of endpoints at available time points. Figure 2 provides visual representation of the association between these combinations of endpoints at the last assessment using bubble plots.

Primary analysis conducted at the last assessment with weighted means of changes from baseline in trough $F_{1}$ and SGRQ showed a large, significant negative correlation coefficient ( $\mathrm{r}$ [95\% CI], N), corresponding to 


\section{Table 3. Number of Study Treatment Groups With a Combination of PRO and Lung Volume Measurement Results}

$\begin{array}{ll} & \text { Lung Volume Measurements } \\ \text { PRO } & \text { Trough FEV }_{1} \quad \text { Inspiratory Total } \\ & \mathrm{FEV}_{1} \mathrm{AUC}_{0-12 \mathrm{~h}} \text { Capacity }\end{array}$

Last Assessment

\begin{tabular}{l|c|c|c|c}
\hline SGRQ & 111 & 22 & 13 & 116 \\
\hline TDI & 68 & 8 & 3 & 68 \\
\hline Annual Exacerbation Rate (overall) & 24 & 0 & 2 & 24 \\
\hline Moderate/Severe Annual Exacerbation Rate & 69 & 9 & 6 & 69 \\
\hline Severe Annual Exacerbation Rate & 6 & 0 & 0 & 6 \\
\hline At 6 months Follow-up & & & & \\
\hline SGRQ & 73 & 18 & 7 & 80 \\
\hline TDI & 52 & 8 & 3 & 54 \\
\hline Annual Exacerbation Rate (overall) & 6 & 0 & 0 & 6 \\
\hline Moderate/Severe Annual Exacerbation Rate & 20 & 5 & 0 & 20 \\
\hline Severe Annual Exacerbation Rate & 0 & 0 & 0 & 0 \\
\hline At 12 months Follow-up & & & & \\
\hline SGRQ & 62 & 4 & 10 & 62 \\
\hline TDI & 20 & 0 & 0 & 20 \\
\hline Annual Exacerbation Rate (overall) & 18 & 0 & 2 & 18 \\
\hline Moderate/Severe Annual Exacerbation Rate & 49 & 4 & 6 & 49 \\
\hline Severe Annual Exacerbation Rate & 6 & 0 & 0 & 6 \\
\hline
\end{tabular}

-0.68 ([-0.77, -0.57], 111) (Table 4). The regression results (weighted) confirmed this highly significant association $(p<0.0001)$ with an improvement of $100 \mathrm{~mL}$ in trough $\mathrm{FEV}_{1}$ corresponding to a reduction of 5.9 in SGRQ total score and a reduction of 4 units on the SGRQ total score, equating to a $40 \mathrm{~mL}$ improvement in trough FEV 1 (Table 4, Figure 2A). Results of weighted analyses between trough $\mathrm{FEV}_{1}$ and the TDI score at the last assessment showed a large, significant positive correlation, with an improvement of $100 \mathrm{~mL}$ in trough $\mathrm{FEV}_{1}$ corresponding to an improvement of 1.9 on the TDI score, while an improvement of 1 point on TDI was equivalent to a $48 \mathrm{~mL}$ reduction in trough $\mathrm{FEV}_{1}$ $(p<0.0001)$ (Table 4, Figure 2B). A large, negative correlation coefficient was obtained using the timeadjusted $\mathrm{FEV}_{1} \mathrm{AUC}_{0-12 \mathrm{~h}}$ and SGRQ at the last assessment. Weighted regression results also indicated a highly significant association ( $p=0.0031)$ between FEV $A_{1} \mathrm{AUC}_{0}$ $12 \mathrm{~h}$ and $\mathrm{SGRQ}$ at last assessment, with an improvement of $100 \mathrm{~mL}$ in $\mathrm{FEV}_{1} \mathrm{AUC}_{0-12 \mathrm{~h}}$ corresponding to an improvement of -5.75 on SGRQ, while an improvement of 4 units on SGRQ corresponds to a $10 \mathrm{~mL}$ reduction in $\mathrm{FEV}_{1} \mathrm{AUC}_{0-12 \mathrm{~h}}$ (Table 4, Figure 2C). Statistically significant negative correlations were obtained between trough $\mathrm{FEV}_{1}$ and the annual rate of exacerbations (overall, moderate or severe). Table 4 and Figures $2 \mathrm{D}$ and $2 \mathrm{E}$ show that improvement in $\mathrm{FEV}_{1}$ leads to reduction in the annual rate of exacerbations. An improvement of $100 \mathrm{~mL}$ in trough $\mathrm{FEV}_{1}$ corresponds to an annual rate of exacerbations of 0.5, while no change on $\mathrm{FEV}_{1}$ corresponds to an annual rate of exacerbations of $2.3(p=0.0002)$. An improvement of $100 \mathrm{~mL}$ in trough $\mathrm{FEV}_{1}$ corresponds to an annual rate of moderate or severe exacerbations of 0.7 , while no change on $\mathrm{FEV}_{1}$ corresponds to an annual rate of moderate or severe exacerbations of $0.9(p<0.0001)$.

Results of the sensitivity analyses conducted at other time points ( 6 and/ or 12 months, subject to availability of data, Table 4) were comparable. Results of the unweighted analyses (Table 4) also were consistent with the results of the weighted analyses.

Further analyses conducted at the last assessment excluding the placebo groups are shown in Table 5. The weighted correlation coefficients at last assessment for the following pairs, trough $\mathrm{FEV}_{1}$ and SGRQ (-0.63), trough FEV 1 and TDI (0.31), $\mathrm{FEV}_{1} \mathrm{AUC}_{0-12 \mathrm{~h}}$ and SGRQ (-0.49), exacerbation rate (overall) and trough $\mathrm{FEV}_{1}(-0.88)$ and exacerbation rate (moderate/severe) and trough $\mathrm{FEV}_{1}(-0.67)$ were statistically significant (all $p<0.05)$ (Table 5, Figure 3). Overall, these results limited to active treatment were similar to the main analysis. The correlations of $\mathrm{FEV}_{1}$ with PROs were significant although slightly decreased; correlations with exacerbation rates were significant and slightly increased.

Further analyses conducted at the last assessment with weighted means of difference over placebo in trough FEV 1 and in SGRQ showed a medium and statistically significant correlation coefficient -0.35 [(-0.56, -0.08), 53] (Table 6). The weighted regression results indicate a significant association between the change beyond placebo in trough $\mathrm{FEV}_{1}$ and in SGRQ at the last assessment $(p<0.05)$, with an improvement over placebo of $100 \mathrm{~mL}$ in trough $\mathrm{FEV}_{1}$ corresponding to a reduction of 2.9 in SGRQ total score and conversely, 


\section{Table 4. Weighted/Unweighted Correlation and Regression Analyses Between FEV 1 CFB and PROs (for Endpoint Combinations Including $\geq 15$ Treatment-Groups)}

\begin{tabular}{|c|c|c|c|c|c|c|c|c|c|}
\hline \multirow[b]{2}{*}{$\mathrm{FEV}_{1}$} & \multirow[b]{2}{*}{ PRO } & \multirow[b]{2}{*}{$\begin{array}{c}\text { Time } \\
\text { Point/ } \\
\text { Severity }\end{array}$} & \multirow[b]{2}{*}{$\mathbf{N}$} & \multicolumn{3}{|c|}{ Weighted Analyses } & \multicolumn{3}{|c|}{ Unweighted Analyses } \\
\hline & & & & $\begin{array}{c}\text { Pearson } \\
\text { Correlation } \\
\text { Coefficient } \\
(95 \% \mathrm{CI})\end{array}$ & $\begin{array}{l}\text { FEV }_{1} \\
\text { Value } \\
\text { Corres- } \\
\text { ponding } \\
\text { to MCID } \\
\text { Value } \\
\text { for PRO }\end{array}$ & $\begin{array}{l}\text { PRO Value } \\
\text { Corres- } \\
\text { ponding } \\
\text { to MCID } \\
\left(0^{a}\right) \text { Value } \\
\text { for FEV }\end{array}$ & $\begin{array}{l}\text { Pearson } \\
\text { Correlation } \\
\text { Coefficient } \\
(95 \% \mathrm{CI})\end{array}$ & $\begin{array}{c}\text { FEV } \\
\text { Value } \\
\text { Corres- } \\
\text { ponding } \\
\text { to MCID } \\
\text { Value } \\
\text { for PRO }\end{array}$ & $\begin{array}{l}\text { PRO Value } \\
\text { Corres- } \\
\text { ponding } \\
\text { to MCID } \\
\left(0^{\mathrm{a})} \text { Value }\right. \\
\text { for } \mathrm{FEV} \mathrm{V}_{1}\end{array}$ \\
\hline \multirow[t]{4}{*}{$\begin{array}{l}\text { Trough } \\
\mathrm{FEV}_{1} \mathrm{CFB}\end{array}$} & $\begin{array}{l}\text { SGRQ } \\
\text { CFB }\end{array}$ & & & & & & & & \\
\hline & & $\begin{array}{c}\text { Last } \\
\text { Assessment }\end{array}$ & 111 & $\begin{array}{c}-0.68 \\
(-0.77,-0.57)\end{array}$ & 40.35 & -5.89 & $\begin{array}{c}-0.63 \\
(-0.73,-0.51)\end{array}$ & 33.79 & -6.01 \\
\hline & & 6 months & 73 & $\begin{array}{c}-0.60 \\
(-0.73,-0.43)\end{array}$ & 30.11 & -5.84 & $\begin{array}{c}-0.51 \\
(-0.66,-0.32)\end{array}$ & 21.40 & -5.82 \\
\hline & & 12 months & 62 & $\begin{array}{c}-0.79 \\
(-0.87,-0.68)\end{array}$ & 52.39 & -5.58 & $\begin{array}{c}-0.78 \\
(-0.86,-0.65)\end{array}$ & 45.63 & -5.91 \\
\hline
\end{tabular}

Trough TDI

$\mathrm{FEV}_{1} \mathrm{CFB}$

\begin{tabular}{l|c|c|c|c|c|c|c|c|c}
\hline & $\begin{array}{c}\text { Last } \\
\text { Assessment }\end{array}$ & 68 & $\begin{array}{c}0.57 \\
(0.38,0.71)\end{array}$ & -47.93 & 1.88 & $\begin{array}{c}0.58 \\
(0.39,0.72)\end{array}$ & -21.98 & 1.77 \\
\hline & 6 months & 52 & $\begin{array}{c}0.55 \\
(0.33,0.72)\end{array}$ & -32.64 & 1.80 & $\begin{array}{c}0.57 \\
(0.36,0.73)\end{array}$ & -10.14 & 1.70 \\
\hline & 12 months & 20 & $\begin{array}{c}0.59 \\
(0.20,0.82)\end{array}$ & -35.92 & 1.91 & $\begin{array}{c}0.61 \\
(0.23,0.83)\end{array}$ & -10.89 & 1.86 \\
\hline
\end{tabular}

Trough Exacerbation

$\mathrm{FEV}_{1} \mathrm{CFB}$ Rate

\begin{tabular}{l|l|c|c|c|c|c|c|c}
\hline & Overall & 24 & $\begin{array}{c}-0.69 \\
(-0.85,-0.39)\end{array}$ & NA & $0.49(2.30)$ & $\begin{array}{c}-0.60 \\
(-0.81,-0.26)\end{array}$ & NA & $\begin{array}{c}0.65 \\
(2.27)\end{array}$ \\
\hline & $\begin{array}{l}\text { Moderate } \\
\text { or Severe }\end{array}$ & 69 & $\begin{array}{c}-0.57 \\
(-0.71,-0.39)\end{array}$ & NA & $0.66(0.94)$ & -0.55 & NA & 0.63 \\
$(-0.70,-0.36)$ & & $(0.90)$ \\
\hline
\end{tabular}

$\mathrm{FEV}_{1} \quad$ SGRQ

$\mathrm{AUC}_{0-12 \mathrm{~h}} \mathrm{CFB}$

$\mathrm{CFB}$

\begin{tabular}{|c|c|c|c|c|c|c|c|}
$\begin{array}{c}\text { Last } \\
\text { Assessment }\end{array}$ & 22 & $\begin{array}{c}-0.60 \\
(-0.82,-0.24)\end{array}$ & -9.76 & -5.75 & $\begin{array}{c}-0.60 \\
(-0.82,-0.25)\end{array}$ & -27.05 & -5.90 \\
\hline 6 months & 18 & $\begin{array}{c}-0.80 \\
(-0.92,-0.54)\end{array}$ & -55.65 & -6.76 & $\begin{array}{c}-0.77 \\
(-0.91,-0.47)\end{array}$ & -47.08 & -6.56 \\
\hline
\end{tabular}

Summary of correspondence of trough FEV ${ }_{1}$ CFB to PROs and PROs to trough FEV 1 CFB for established MCIDs (-4 for SGRQ, +1 for TDI and $100 \mathrm{~mL}$ for $\mathrm{FEV}_{1}$ ) in the simple weighted/unweighted linear regression models of PROs (y) on $\mathrm{FEV}_{1}(\mathrm{x})$, for plots including at least 15 dots.

${ }^{a}$ The correspondence to a 0 change in $\mathrm{FEV}_{1}$ is calculated to facilitate interpretation of exacerbation rates corresponding to $\mathrm{FEV}_{1} \mathrm{MCID}$.

$\mathrm{N}$, number of dots in the bubble plot, i.e., the number of study treatment groups with both endpoints $\mathrm{x}$ and $\mathrm{y}$ available at the same time point and used to estimate the parameters of the linear regression of $\mathrm{y}$ on $\mathrm{x}$;

NA, not applicable as analysis not conducted due to sample size $<15$ as specified in the Statistical Analysis Plan. 


\section{Table 5. Weighted/Unweighted Correlation and Regression Analyses for FEV 1 CFB and PROs (Last Assessment) Excluding Placebo Groups}

\begin{tabular}{|c|c|c|c|c|c|c|c|c|}
\hline \multirow[b]{2}{*}{$\mathrm{FEV}_{1}$} & \multirow[b]{2}{*}{ PRO } & \multirow[b]{2}{*}{$\mathbf{N}$} & \multicolumn{3}{|c|}{ Weighted Analyses } & \multicolumn{3}{|c|}{ Unweighted Analyses } \\
\hline & & & $\begin{array}{l}\text { Pearson } \\
\text { Correlation } \\
\text { Coefficient } \\
(95 \% \mathrm{CI})\end{array}$ & $\begin{array}{l}\text { FEV } 1 \\
\text { Value } \\
\text { Corres- } \\
\text { ponding } \\
\text { to MCID } \\
\text { Value } \\
\text { for PRO }\end{array}$ & $\begin{array}{l}\text { PRO Value } \\
\text { Corres- } \\
\text { ponding } \\
\text { to MCID } \\
\left(0^{a}\right) \text { Value } \\
\text { for FEV } 1\end{array}$ & $\begin{array}{c}\text { Pearson } \\
\text { Correlation } \\
\text { Coefficient } \\
(95 \% \mathrm{CI})\end{array}$ & $\begin{array}{c}\text { FEV } 1 \\
\text { Value } \\
\text { Corres- } \\
\text { ponding } \\
\text { to MCID } \\
\text { Value } \\
\text { for PRO }\end{array}$ & $\begin{array}{l}\text { PRO Value } \\
\text { Corres- } \\
\text { ponding } \\
\text { to MCID } \\
\left(0^{a}\right) \text { Value } \\
\text { for FEV } 1\end{array}$ \\
\hline $\begin{array}{l}\text { Trough FEV } 1 \\
\text { CFB (mL) }\end{array}$ & SGRQ CFB & 86 & $\begin{array}{r}-0.63 \\
(-0.75,-0.49)\end{array}$ & 40.79 & -5.98 & $\begin{array}{r}-0.62 \\
(-0.73,-0.46)\end{array}$ & 34.10 & -6.14 \\
\hline $\begin{array}{l}\text { Trough FEV } 1 \\
\text { CFB }(\mathrm{mL})\end{array}$ & TDI & 52 & $\begin{array}{r}0.31 \\
(0.04,0.54)\end{array}$ & -143.32 & 1.90 & $\begin{array}{r}0.39 \\
(0.13,0.60)\end{array}$ & -49.68 & 1.79 \\
\hline $\begin{array}{l}\text { Trough FEV } 1 \\
\text { CFB }(\mathrm{mL})\end{array}$ & $\begin{array}{l}\text { Annual Exacerbation } \\
\text { Rate (overall) }\end{array}$ & 19 & $\begin{array}{r}-0.88 \\
(-0.95,-0.70)\end{array}$ & NA & $\begin{array}{l}0.33 \\
(2.99)\end{array}$ & $\begin{array}{r}-0.79 \\
(-0.92,-0.53)\end{array}$ & NA & $\begin{array}{l}0.47 \\
(2.80)\end{array}$ \\
\hline $\begin{array}{l}\text { Trough FEV } 1 \\
\text { CFB (mL) }\end{array}$ & $\begin{array}{l}\text { Moderate/Severe } \\
\text { Annual Exacerbation } \\
\text { Rate }\end{array}$ & 53 & $\begin{array}{r}-0.67 \\
(-0.80,-0.49)\end{array}$ & NA & $\begin{array}{c}0.66 \\
(0.97)\end{array}$ & $\begin{array}{r}-0.72 \\
(-0.83,-0.55)\end{array}$ & NA & $\begin{array}{c}0.61 \\
(0.98)\end{array}$ \\
\hline $\begin{array}{l}\mathrm{FEV}_{1} \mathrm{AUC}_{0-12 \mathrm{~h}} \\
\text { CFB (mL) }\end{array}$ & SGRQ CFB & 17 & $\begin{array}{r}-0.49 \\
(-0.79,-0.02)\end{array}$ & -43.61 & -6.01 & $\begin{array}{r}-0.53 \\
(-0.81,-0.07)\end{array}$ & -54.90 & -6.12 \\
\hline
\end{tabular}

Weighted and unweighted Pearson correlation coefficients and linear regression results for different combinations of spirometric measurements and PROs at the last assessment excluding placebo group results.

${ }^{\mathrm{a}}$ The correspondence to a 0 change in $\mathrm{FEV}_{1}$ is calculated to facilitate interpretation of exacerbation rates corresponding to $\mathrm{FEV}_{1} \mathrm{MCID}$

$\mathrm{N}$, number of dots in the bubble plot, i.e., the number of study treatment groups with both endpoints $\mathrm{x}$ and $\mathrm{y}$ available at the same time point and used to estimate the parameters of the linear regression of $\mathrm{y}$ on $\mathrm{x}$;

NA, not applicable as analysis not conducted due to sample size $<15$ as specified in the Statistical Analysis Plan.

a reduction of 4 units on the SGRQ total score, corresponding to a $201 \mathrm{~mL}$ improvement in trough $\mathrm{FEV}_{1}$ beyond placebo (Table 6, Figure 4). Analysis of all other combinations of endpoints exploring the association of effects beyond placebo on $\mathrm{FEV}_{1}$ and on PROs, with weighted or unweighted approach (Table 6) lead to nonsignificant results $(p>0.05)$.

\section{Discussion}

Both objectively measured lung function and subjectively measured $\mathrm{PRO}$ s are frequently assessed during COPD clinical management. Both of these endpoints remain important to decision makers with regulators preferring to assess benefits of new treatments on lung function and payers on PROs. However, data on the association between spirometric measurements and PROs among patients with COPD are sparse, generally limited to a single study context and with different methodologies and outcomes potentially leading to variable conclusions. ${ }^{63-66}$ A previous meta-analysis ${ }^{67}$ evaluated the association between lung function measurements and PROs in bronchodilator trials. This study further explores the relationship between spirometric measurements and PROs and includes current evidence from combination therapies in COPD trials.

Our primary analysis showed a large and highly significant association between SGRQ and trough $\mathrm{FEV}_{1}$. Analyses with other pairings of spirometric measurements and PROs showed correspondingly large correlation coefficients, and a similar trend: A MCID change in $\mathrm{FEV}_{1}$ corresponding to a larger than MCID change in PROs. Such trends, where significant changes in $\mathrm{PROs}$ are associated with subclinical changes in objective parameters (such as $\mathrm{FEV}_{1}$ ), are often encountered in clinical trials. Potential contributing factors to this phenomenon are 


\section{Table 6. Weighted/Unweighted Correlation and Regression Analyses for FEV 1 CFB and PROs (Last Assessment) Beyond Placebo Effect}

\begin{tabular}{|c|c|c|c|c|c|c|c|c|}
\hline \multirow[b]{2}{*}{$\mathrm{FEV}_{1}$} & \multirow[b]{2}{*}{ PRO } & \multirow[b]{2}{*}{$\mathbf{N}$} & \multicolumn{3}{|c|}{ Weighted Analyses } & \multicolumn{3}{|c|}{ Unweighted Analyses } \\
\hline & & & $\begin{array}{l}\text { Pearson } \\
\text { Correlation } \\
\text { Coefficient } \\
(95 \% \mathrm{Cl})\end{array}$ & $\begin{array}{l}\text { FEV } 1 \\
\text { Value } \\
\text { Corres- } \\
\text { ponding } \\
\text { to MCID } \\
\text { Value } \\
\text { for PRO }\end{array}$ & $\begin{array}{l}\text { PRO Value } \\
\text { Corres- } \\
\text { ponding } \\
\text { to MCID } \\
\left(0^{2}\right) \text { Value } \\
\text { for FEV }\end{array}$ & $\begin{array}{l}\text { Pearson } \\
\text { Correlation } \\
\text { Coefficient } \\
(95 \% \mathrm{CI})\end{array}$ & $\begin{array}{l}\text { FEV }_{1} \\
\text { Value } \\
\text { Corres- } \\
\text { ponding } \\
\text { to MCID } \\
\text { Value } \\
\text { for } Y\end{array}$ & $\begin{array}{l}\text { PRO Value } \\
\text { Corres- } \\
\text { ponding } \\
\text { to MCID } \\
\left(0^{a}\right) \text { Value } \\
\text { for } \mathrm{FEV}_{1}\end{array}$ \\
\hline $\begin{array}{l}\text { Difference in } \\
\text { Mean CFB } \\
\text { Trough FEV } 1 \\
\text { (mL) }\end{array}$ & $\begin{array}{l}\text { Difference in Mean } \\
\text { CFB SGRQ }\end{array}$ & 53 & $\begin{array}{r}-0.35 \\
(-0.56,-0.08)\end{array}$ & 200.50 & -2.93 & $\begin{array}{r}-0.00 \\
(-0.27,0.27)\end{array}$ & 12477.1 & -3.02 \\
\hline $\begin{array}{l}\text { Difference in } \\
\text { Mean CFB } \\
\text { Trough FEV }{ }_{1} \\
(\mathrm{~mL})\end{array}$ & Difference in Mean TDI & 38 & $\begin{array}{r}0.24 \\
(-0.08,0.52)\end{array}$ & 244.35 & 0.83 & $\begin{array}{r}0.30 \\
(-0.02,0.56)\end{array}$ & 219.66 & 0.80 \\
\hline $\begin{array}{l}\text { Difference in } \\
\text { Mean CFB } \\
\text { Trough FEV } \\
(\mathrm{mL})\end{array}$ & $\begin{array}{l}\text { Difference in Moderate/ } \\
\text { Severe Annual } \\
\text { Exacerbation Rate }\end{array}$ & 33 & $\begin{array}{r}-0.19 \\
(-0.50,0.16)\end{array}$ & NA & $\begin{array}{r}-0.26 \\
(-0.17)\end{array}$ & $\begin{array}{r}-0.13 \\
(-0.45,0.22)\end{array}$ & NA & $\begin{array}{r}-0.25 \\
(-0.20)\end{array}$ \\
\hline $\begin{array}{l}\text { Difference in } \\
\text { Mean FEV }_{1} \\
\text { AUC }_{0-12 \mathrm{~h}} \\
\text { CFB (mL) }\end{array}$ & $\begin{array}{l}\text { Difference in Mean } \\
\text { CFB SGRQ }\end{array}$ & 17 & $\begin{array}{r}-0.10 \\
(-0.56,0.40)\end{array}$ & 1305.93 & -2.68 & $\begin{array}{r}-0.07 \\
(-0.53,0.42)\end{array}$ & 1477.39 & -2.86 \\
\hline
\end{tabular}

Weighted and unweighted Pearson correlation coefficients and linear regression results for different combinations of spirometric measurements and patient reported outcomes at the last assessment of the differences beyond the placebo effect.

${ }^{\mathrm{a}}$ The correspondence to a 0 change in $\mathrm{FEV}_{1}$ is calculated to facilitate interpretation of exacerbation rates corresponding to $\mathrm{FEV} \mathrm{MCID}_{1}$

$\mathrm{N}$, number of treatment groups included in the calculation of the weighted Pearson correlation coeefficient;

NA, not applicable as analysis not conducted due to sample size $<15$ as specified in the Statistical Analysis Plan.

the Hawthorne effect, wherein the study participants change their behavior because they are observed, or the Pygmalion effect whereby the patients' desire to meet the expectations of their clinician or the study sponsor tends to exaggerate their symptoms and their impacts at inclusion and minimize these at follow-up, ${ }^{68}$ leading to optimistic change over time. As these factors are observed in both active and placebo arms, there are no consequences for treatment group comparisons, though the phenomena may result in apparent discrepancies in MCID values and regression estimates for subjective and objective measurements, as observed in the present study. Further, it must be considered that as each
MCID has been established independently and using different methods, ${ }^{8-10}$ it is therefore not surprising to obtain results that do not match. Result of our analyses on combination therapies including newly launched combination bronchodilators, provides a more comprehensive meta-analysis (52 studies; 62,385 patients versus 22 studies; 23,654 patients) compared to the Westwood et al analysis. ${ }^{67}$ The results of the analysis at 6 and 12 months' follow-up suggest that the correlation of trough FEV 1 with SGRQ and TDI strengthens with time, consistent with the previous study. ${ }^{67}$ This association decreased slightly after removal of the placebo groups from the analysis and 


\section{Figure 2. Association Between FEV 1 and PROs at the Last Assessment}
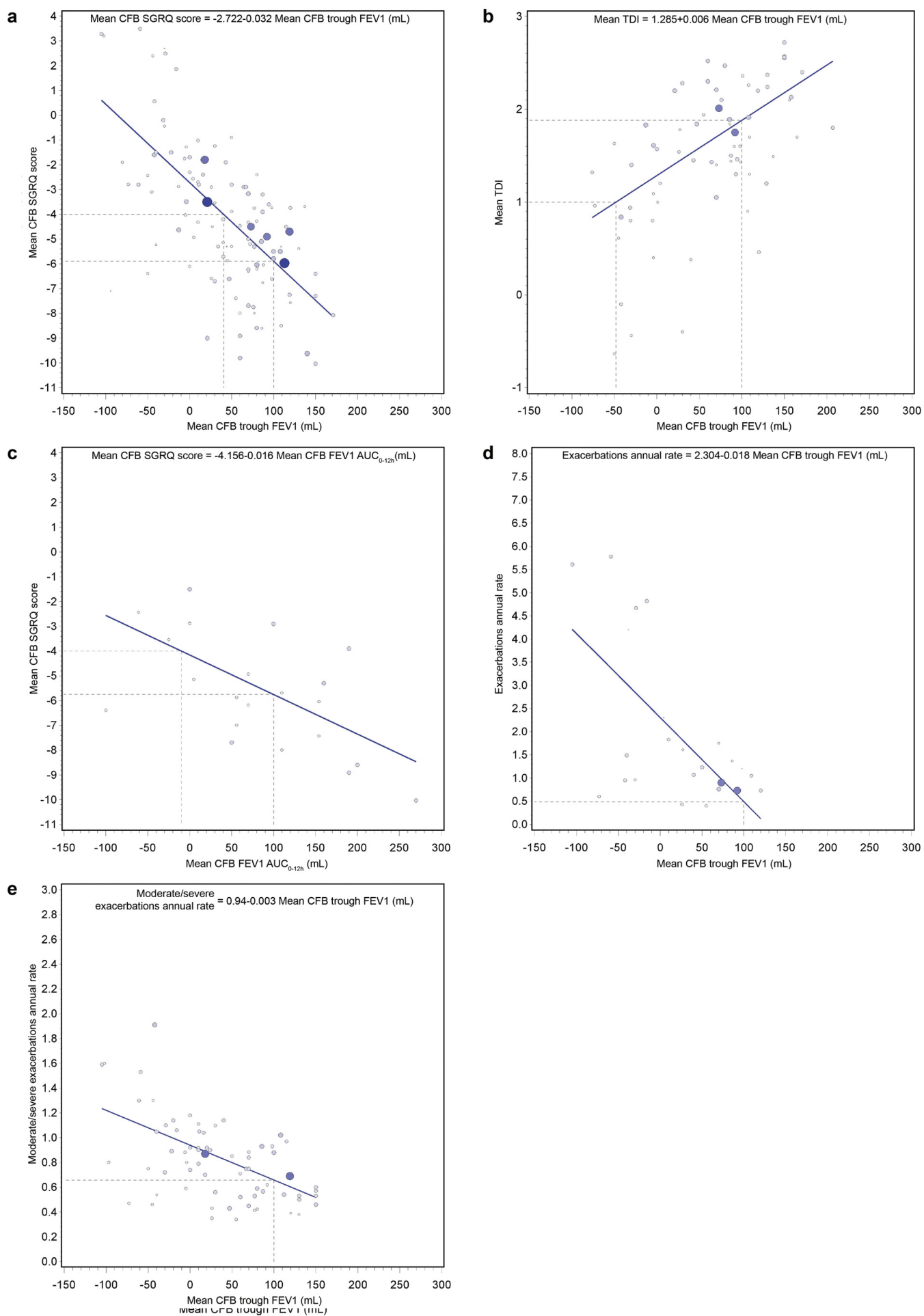

Each dot of these bubble plots represents a study treatment-group result, with coordinate $\mathrm{x}$ being the mean spirometric measurement $\mathrm{CFB}$, and coordinate y being the mean PRO; the size of the dot is proportional to the sample size of the study treatment group over the total number of patients across all studies. The equation of the line drawn was estimated using a simple weighted regression model.

2A: Mean SGRQ CFB by mean trough $\mathrm{FEV}_{1} \mathrm{CFB}$ at last assessment $(\mathrm{N}=111)$

2B: Mean TDI by mean trough $\mathrm{FEV}_{1} \mathrm{CFB}$ at last assessment $(\mathrm{N}=111)$

2C: Mean SGRQ CFB by mean $\mathrm{FEV}_{1} \mathrm{AUC}_{0-12}$ hours CFB at last assessment $(\mathrm{N}=22)$

2D: Annual rate of exacerbations by mean trough $\mathrm{FEV} \mathrm{C}_{1} \mathrm{CFB}(\mathrm{N}=24)$

2E: Annual rate of moderate or severe exacerbations by mean trough $\mathrm{FEV}_{1} \mathrm{CFB}(\mathrm{N}=69)$ 


\section{Figure 3. Association Between FEV 1 and PROs at Last Assessment; Analyses Limited to Results Excluding Placebo Groups}
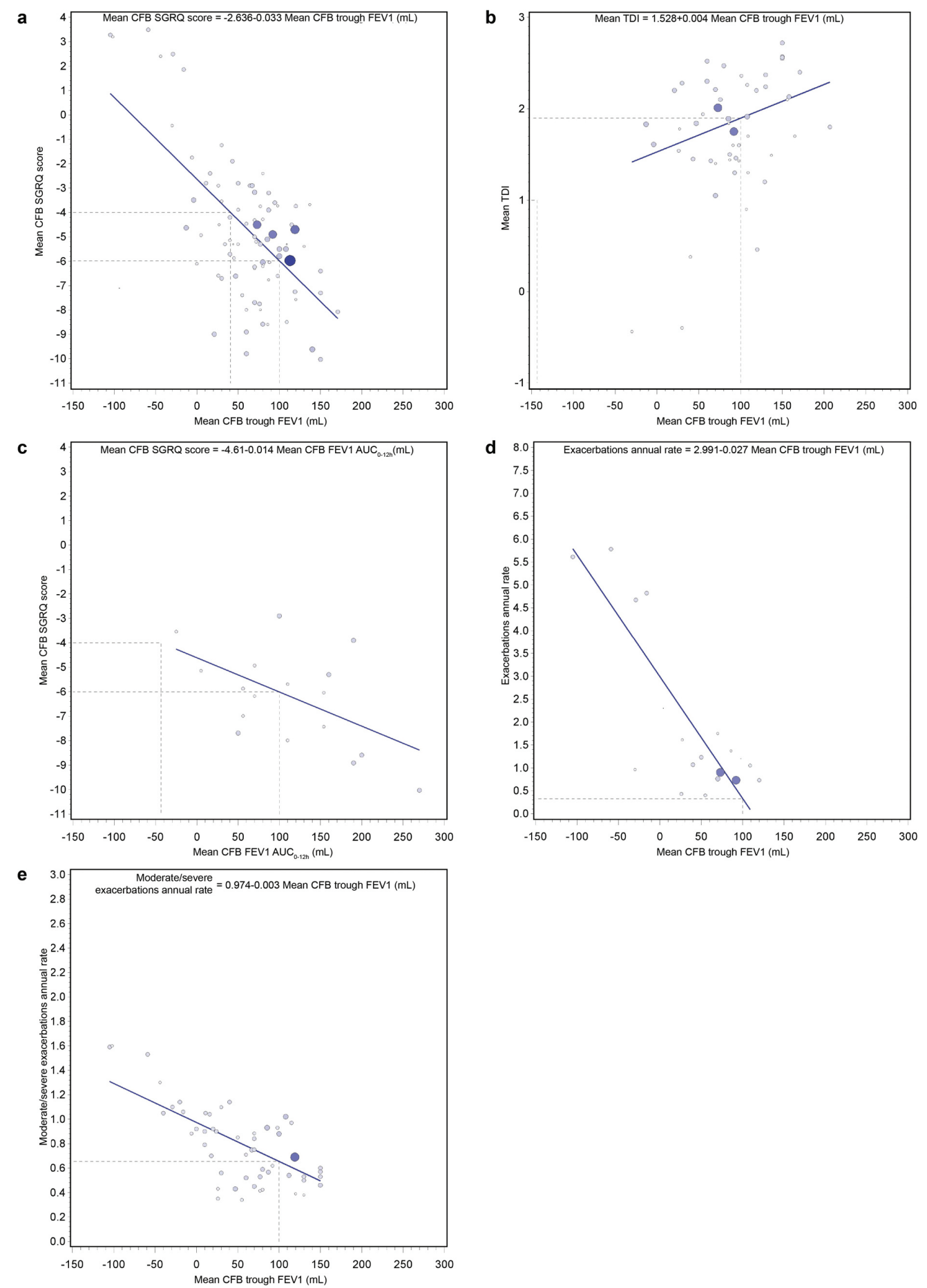

Each dot of these bubble plots represents a study treatment-group result, with coordinate $\mathrm{x}$ being the mean spirometric measurement CFB, and coordinate y being the mean PRO; the size of the dot is proportional to the sample size of the study treatment group over the total number of patients across all studies. The equation of the line drawn was estimated using a simple weighted regression model.

3A: Mean SGRQ CFB by mean trough $\mathrm{FEV}_{1} \mathrm{CFB}$ at last assessment $(\mathrm{N}=111)$

3B: Mean TDI by mean trough $\mathrm{FEV}_{1} \mathrm{CFB}$ at last assessment $(\mathrm{N}=111)$

3C: Mean SGRQ CFB by mean $\mathrm{FEV}_{1} \mathrm{AUC}_{0-12}$ hours CFB at last assessment $(\mathrm{N}=22)$

3D: Annual rate of exacerbations by mean trough $\mathrm{FEV} \mathrm{V}_{1} \mathrm{CFB}(\mathrm{N}=24)$

3E: Annual rate of moderate or severe exacerbations by mean trough $\mathrm{FEV}_{1} \mathrm{CFB}(\mathrm{N}=69)$ 


\section{Figure 4. Association Between Difference Over Placebo in FEV 1 CFB and in PROs at Last Assessment}

a

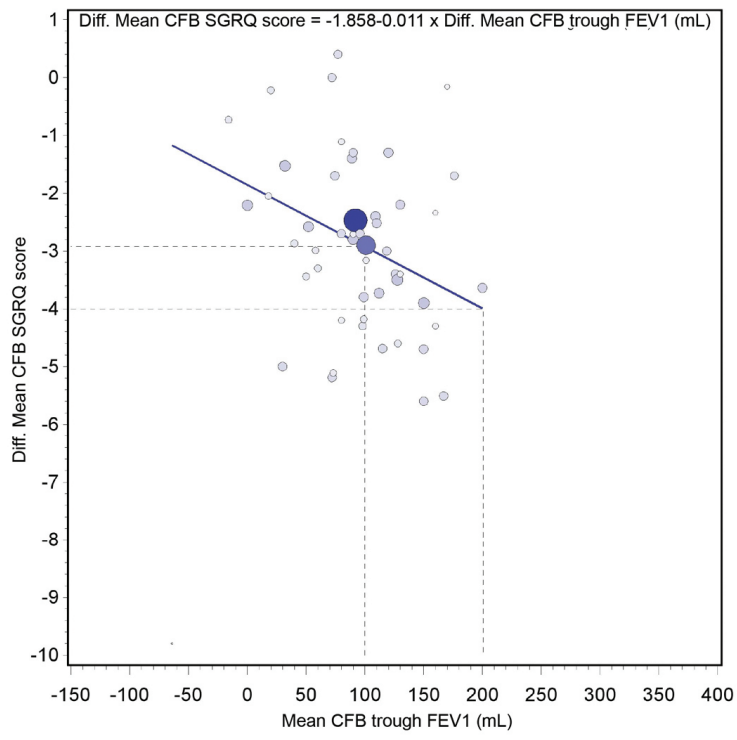

C

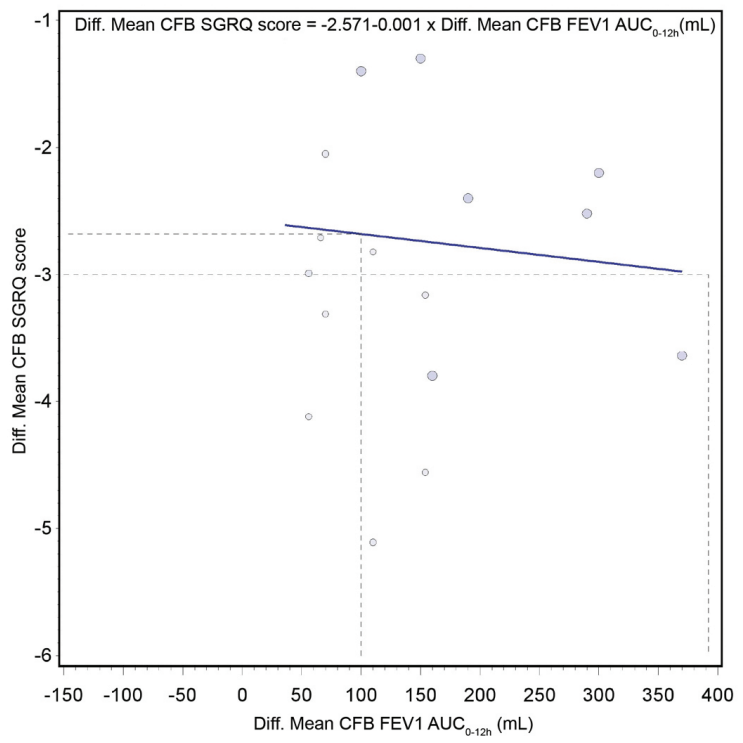

b

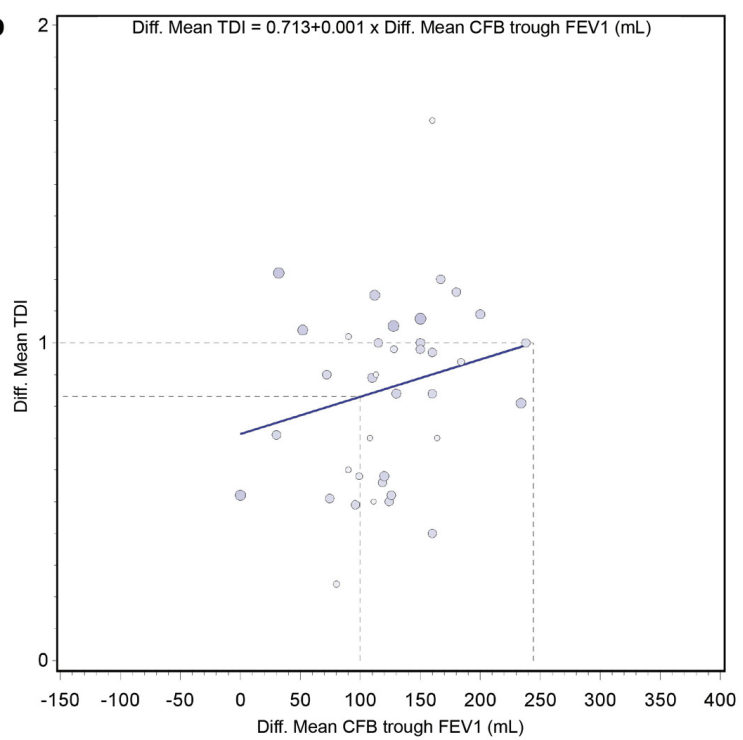

d

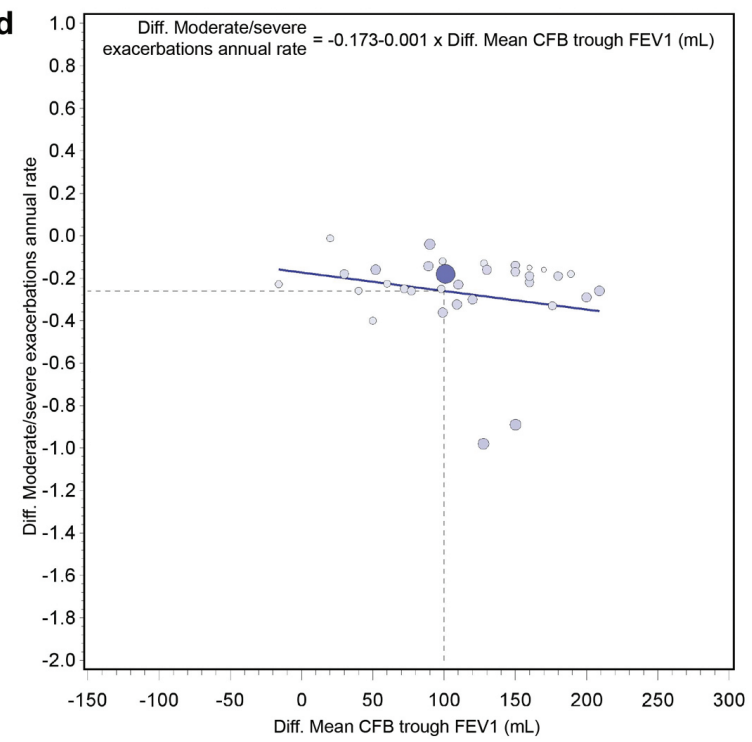

Each dot of these bubble plots represents a study treatment group results with coordinate $\mathrm{x}$ being the difference in mean spirometric measurement CFB for the active treatment group minus mean results obtained for the placebo group, and coordinate y, the difference in mean PRO obtained for the active treatment group minus mean result obtained in the placebo group; the size of the dot is proportional to the sample size of the study treatment group over the total number of patients across all studies. The equation of the line drawn was estimated in a simple weighted regression model.

4A: Mean SGRQ CFB by mean trough $\mathrm{FEV}_{1} \mathrm{CFB}(\mathrm{N}=53)$

4B: Mean TDI by mean trough $\mathrm{FEV}_{1} \mathrm{CFB}(\mathrm{N}=38)$

4C: Mean SGRQ CFB by mean $\mathrm{FEV}_{1} \mathrm{AUC}_{0-12}$ hours $\mathrm{CFB}(\mathrm{N}=17)$

4D: Annual rate of moderate or severe exacerbations by mean trough $\mathrm{FEV}_{1} \mathrm{CFB}(\mathrm{N}=33)$

decreased largely when analyzing treatment effects beyond placebo. The association between FEV 1 and SGRQ however, remained significant. Overall, the results were consistent with the Westwood et al study suggesting that the association between trough $\mathrm{FEV}_{1}$ and $\mathrm{PROs}$ observed in bronchodilator studies remains with combination therapies.

Results of the analysis exploring the association of treatment effects beyond placebo are of particular interest. The correlation between $\mathrm{FEV}_{1}$ and SGRQ at last assessment was significant while all other associations did not reach statistical significance. 
Corresponding regression results indicated that an improvement of $100 \mathrm{~mL}$ over placebo in trough $\mathrm{FEV}_{1}$ corresponds to a reduction of 2.9 in SGRQ total score and conversely, a reduction of 4 units in the SGRQ total score corresponds to a $201 \mathrm{~mL}$ improvement in trough $\mathrm{FEV}_{1}$ beyond placebo. These estimates are broadly consistent with the results observed in recent studies of dual bronchodilators ${ }^{17,69}$ and indicate that after eliminating the placebo effect, a 4 point (MCID) change difference on the SGRQ score represents a much larger change than the 100mL MCID for FEV 1 . It must be noted that these analyses beyond placebo effect excluded 17 clinical trials that were not placebocontrolled-generally conducted in patients with more severe disease-which may have led to a selection bias. Limiting the analysis to more severe disease with limited variability is particularly detrimental to regression analyses. Further research is needed to address this conclusively.

Some limitations of our meta-analysis must be acknowledged. Given the unavailability of individualpatient data, the meta-analysis was conducted using study-level data and the precision of the results would have been increased if the individual-patient data were available. Although we conducted an extensive search of the clinical trial registries and websites of the regulatory authorities to minimize publication bias, this meta-analysis is still limited by the availability of data in the public domain. Furthermore, not all endpoints of interest are available for all studies and also, the endpoint definitions may differ between studies especially for variables such as exacerbation rate and severity of exacerbation. However, given the rigorous methodology followed while ascertaining the endpoint definitions for each study, the risk of misclassification should be minimal. As the studies included are clinical trials of bronchodilators, the study populations for these trials do not usually include an exacerbating patient population, which may lead to fewer exacerbations in these trials. Furthermore, exacerbations are included as safety rather than efficacy endpoints. Thus, these trials are not powered to assess differences in exacerbation rates of the study groups, which would affect the

\section{Conclusions}

The results of this meta-analysis provide important clinically meaningful insights into the relationship between $\mathrm{FEV}_{1}$, the standard primary endpoint for $\mathrm{COPD}$ clinical trials, and PROs, namely SGRQ health status measure, TDI, and annual exacerbation rates. Besides including additional clinical trials published in the past few years, the study provides results on new endpoints such as the relationship between $\mathrm{FEV}_{1}$ and the annual rate of exacerbations. The strength of these associations is largely decreased when results beyond placebo effect are assessed. Overall, the results of our correlation and regression analyses demonstrate a strong association between changes in spirometric measurements and changes in PROs from their baseline values.

\section{Acknowledgments}

This study was funded by GlaxoSmithKline. All listed authors met the criteria for authorship set forth by the International Committee for Medical Journal Editors. The authors would like to acknowledge Eline Huisman for her technical support in the systematic review and data extraction and Juliette Meunier for her technical support in SAS programming. Medical writing services were provided by Vidula Bhole, MD, MHSc, of Cactus Communications and funded by GlaxoSmithKline.

\section{Declaration of Interest}

YSP, $\mathrm{MH}, \mathrm{M}$ V-B and ASI are employees of GlaxoSmithKline and hold stock in GlaxoSmithKline. UT was an employee of GlaxoSmithKline at the time of this study and held stock in GlaxoSmithKline. BT, FF, and JL are employed by Mapi and were paid consultants to GlaxoSmithKline. CdL works as an independent consultant and was paid by Mapi to participate in this study. All authors contributed to the conception and design of the study. CdL, BT, FF and JL contributed to data acquisition and analysis. All authors contributed to data analysis and interpretation. 


\section{References}

1. Viegi G, Pistelli F, Sherrill DL, et al. Definition, epidemiology and natural history of COPD. Eur Respir J. 2007; 30(5): 993-1013. doi :http://dx.doi.org/10.1183/09031936.00082507

2. Vestbo J, Hurd SS, Agusti AG, et al. Global strategy for the diagnosis, management, and prevention of chronic obstructive pulmonary disease: GOLD executive summary. Am J Respir Crit Care Med. 2013; 187(4): 347-365.

doi: http://dx.doi.org/10.1164/rccm.201204-0596PP

3. Rabe KF, Hurd S, Anzueto A, et al. Global strategy for the diagnosis, management, and prevention of chronic obstructive pulmonary disease: GOLD executive summary. Am J Respir Crit Care Med. 2007; 176(6): 532-555.

doi: http://dx.doi.org/10.1164/rccm.200703-456SO

4. IQWiG: Institute for Quality and Efficiency in Health Care [German]. General Methods Version 4.1. IQWiG website. https://www.iqwig.de/download/IQWiG_General_Methods_ Version_\%204-1.pdf Published November 28, 2013. Accessed February 25, 2016

5. Jones PW, Quirk FH, Baveystock CM, Littlejohns P. A selfcomplete measure of health status for chronic airflow limitation. The St. George's Respiratory Questionnaire. Am Rev Respir Dis. 1992; 145(6): 1321-1327.

doi: http://dx.doi.org/10.1164/ajrccm/145.6.1321

6. Jones PW, Quirk FH, Baveystock CM. The St. George's Respiratory Questionnaire. Respiratory Med. 1991; 85 (Suppl 2): 25-31. doi: http://dx.doi.org/10.1016/S0954-6111(06)80166-6

7. Mahler DA, Weinberg DH, Wells CK, Feinstein AR. The measurement of dyspnea. Contents, interobserver agreement, and physiologic correlates of two new clinical indexes. Chest. 1984;85(6):751-758. doi: http://dx.doi.org/10.1378/chest.85.6.751

8. Jones PW. St. George's Respiratory Questionnaire: MCID. COPD. 2005; 2(1): 75-79.

doi: http://dx.doi.org/10.1081/COPD-200050513

9. Mahler DA, Witek TJ, Jr. The MCID of the transition dyspnea index is a total score of one unit. COPD. 2005; 2(1): 99-103. doi: http://dx.doi.org/10.1081/COPD-200050666

10. Donohue JF. Minimal clinically important differences in COPD lung function. COPD. 2005; 2(1): 111-124.

doi: http://dx.doi.org/10.1081/COPD-200053377

11. Chapman KR, Bergeron C, Bhutani M, et al. Do we know the minimal clinically important difference (MCID) for COPD exacerbations? COPD. 2013; 10(2): 243-249.

doi: http://dx.doi.org/10.3109/15412555.2012.733463

12. Cohen J. Statistical Power Analysis For The Behavioral Sciences. Maywah, New Jersey: Lawrence Erlbaum Associates. 1988.
13. Celli B, Crater G, Kilbride S, et al. Once-daily umeclidinium/ vilanterol 125/25 mcg in COPD: a randomized, controlled study. Chest. 2014; 145(5): 981-991.

doi: http://dx.doi.org/10.1378/chest.13-1579

14. Abrahams R, Moroni-Zentgraf P, Ramsdell J, et al. Safety and efficacy of the once-daily anticholinergic BEA2180 compared with tiotropium in patients with COPD. Respir Med. 2013; 107(6): 854-862. doi: http://dx.doi.org/10.1016/j.rmed.2013.02.005

15. Bateman ED, Ferguson GT, Barnes N, et al. Dual bronchodilation with QVA149 versus single bronchodilator therapy: the SHINE study. Eur Respir J. 2013; 42(6): 1484-1494. doi: http://dx.doi.org/10.1183/09031936.00200212

16. Decramer ML, Chapman KR, Dahl R, et al. Once-daily indacaterol versus tiotropium for patients with severe chronic obstructive pulmonary disease (INVIGORATE): a randomised, blinded, parallel-group study. Lancet Respir Med. 2013; 1(7): 524-533. doi: http://dx.doi.org/10.1016/S2213-2600(13)70158-9

17. Donohue JF, Maleki-Yazdi MR, Kilbride S, et al. Efficacy and safety of once-daily umeclidinium/vilanterol $62.5 / 25 \mathrm{mcg}$ in COPD. Respir Med. 2013; 107(10): 1538-1546. doi: http://dx.doi.org/10.1016/j.rmed.2013.06.001

18. Dransfield MT, Bourbeau J, Jones PW, et al. Once-daily inhaled fluticasone furoate and vilanterol versus vilanterol only for prevention of exacerbations of COPD: two replicate doubleblind, parallel-group, randomised controlled trials. Lancet Respir Med. 2013; 1(3): 210-223.

doi: http://dx.doi.org/10.1016/S2213-2600(13)70040-7

19. D’Urzo A, Kerwin E, Rennard S, et al. One-year extension study of ACCORD COPD I: safety and efficacy of two doses of twicedaily aclidinium bromide in patients with COPD. COPD. 2013; 10(4): 500-510.

doi: http://dx.doi.org/10.3109/15412555.2013.791809

20. Gelb AF, Tashkin DP, Make BJ, et al. Long-term safety and efficacy of twice-daily aclidinium bromide in patients with COPD. Respir Med. 2013; 107(12): 1957-1965.

doi: http://dx.doi.org/10.1016/j.rmed.2013.07.001

21. Kerwin EM, Scott-Wilson C, Sanford L, et al. A randomised trial of fluticasone furoate/vilanterol (50/25 mug; 100/25 mug) on lung function in COPD. Respir Med. 2013; 107(4): 560-569. doi: http://dx.doi.org/10.1016/j.rmed.2012.12.014

22. Martinez FJ, Boscia J, Feldman G, et al. Fluticasone furoate/ vilanterol (100/25; 200/25 mug) improves lung function in COPD: a randomised trial. Respir Med. 2013; 107(4): 550-559. doi: http://dx.doi.org/10.1016/j.rmed.2012.12.016

23. Vogelmeier CF, Bateman ED, Pallante J, et al. Efficacy and safety of once-daily QVA149 compared with twice-daily salmeterolfluticasone in patients with chronic obstructive pulmonary disease (ILLUMINATE): a randomised, double-blind, parallel group study. Lancet Respir Med. 2013; 1(1): 51-60. doi: http://dx.doi.org/10.1016/S2213-2600(12)70052-8 
$\overline{\text { 24. Wedzicha JA, Decramer M, Ficker JH, et al. Analysis of chronic }}$ obstructive pulmonary disease exacerbations with the dual bronchodilator QVA149 compared with glycopyrronium and tiotropium (SPARK): a randomised, double-blind, parallel-group study. Lancet Respir Med. 2013; 1(3): 199-209.

doi: http://dx.doi.org/10.1016/S2213-2600(13)70052-3

25. Doherty DE, Tashkin DP, Kerwin E, et al. Effects of mometasone furoate/formoterol fumarate fixed-dose combination formulation on chronic obstructive pulmonary disease (COPD): results from a 52-week Phase III trial in subjects with moderate-to-very severe COPD. Int J Chron Obstruct Pulmon Dis. 2012; 7:57-71. doi: http://dx.doi.org/10.2147/COPD.S27320

26. Hanania NA, Crater GD, Morris AN, et al. Benefits of adding fluticasone propionate/salmeterol to tiotropium in moderate to severe COPD. Respir Med. 2012; 106(1): 91-101. doi: http://dx.doi.org/10.1016/j.rmed.2011.09.002

27. Jones PW, Singh D, Bateman ED, et al. Efficacy and safety of twice-daily aclidinium bromide in COPD patients: the ATTAIN study. Eur Respir J. 2012; 40(4): 830-836. doi: http://dx.doi.org/10.1183/09031936.00225511

28. Kerwin E, Hebert J, Gallagher N, et al. Efficacy and safety of NVA237 versus placebo and tiotropium in patients with COPD: the GLOW2 study. Eur Respir J. 2012; 40(5): 1106-1114. doi: http://dx.doi.org/10.1183/09031936.00040712

29. Sharafkhaneh A, Southard JG, Goldman M, Uryniak T, Martin UJ. Effect of budesonide/formoterol pMDI on COPD exacerbations: a double-blind, randomized study. Respir Med. 2012; 106(2): 257-268.

doi: http://dx.doi.org/10.1016/j.rmed.2011.07.020

30. Tashkin DP, Doherty DE, Kerwin E, et al. Efficacy and safety of a fixed-dose combination of mometasone furoate and formoterol fumarate in subjects with moderate to very severe COPD: results from a 52-week Phase III trial. Int J Chron Obstruct Pulmon Dis. 2012; 7:43-55.

doi: http://dx.doi.org/10.2147/COPD.S27319

31. Chapman KR, Rennard SI, Dogra A, et al. Long-term safety and efficacy of indacaterol, a long-acting beta(2)-agonist, in subjects with COPD: a randomized, placebo-controlled study. Chest. 2011; 140(1): 68-75. doi: http://dx.doi.org/10.1378/chest.10-1830

32. D’Urzo A, Ferguson GT, van Noord JA, et al. Efficacy and safety of once-daily NVA237 in patients with moderate-to-severe COPD: the GLOW1 trial. Respir Res. 2011; 12:156. doi: http://dx.doi.org/10.1186/1465-9921-12-156

33. Jones PW, Rennard SI, Agusti A, et al. Efficacy and safety of once-daily aclidinium in chronic obstructive pulmonary disease. Respir Res. 2011; 12:55.

doi: http://dx.doi.org/10.1186/1465-9921-12-55

34. Bateman E, Singh D, Smith D, et al. Efficacy and safety of tiotropium Respimat SMI in COPD in two 1-year randomized studies. Int J Chron Obstruct Pulmon Dis. 2010; 5:197-208.
35. Bateman ED, Tashkin D, Siafakas N, et al. A one-year trial of tiotropium Respimat plus usual therapy in COPD patients. Respir Med. 2010; 104(10): 1460-1472. doi: http://dx.doi.org/10.1016/j.rmed.2010.06.004

36. Calverley PM, Kuna P, Monso E, et al. Beclomethasone/ formoterol in the management of COPD: a randomised controlled trial. Respir Med. 2010; 104(12): 1858-1868. doi: http://dx.doi.org/10.1016/j.rmed.2010.09.008

37. Dahl R, Chung KF, Buhl R, et al. Efficacy of a new once-daily long-acting inhaled beta2-agonist indacaterol versus twice-daily formoterol in COPD. Thorax. 2010; 65(6): 473-479. doi: http://dx.doi.org/10.1136/thx.2009.125435

38. Donohue JF, Fogarty C, Lotvall J, et al. Once-daily bronchodilators for chronic obstructive pulmonary disease: indacaterol versus tiotropium. Am J Respir Crit Care Med. 2010; 182(2): 155-162. doi: http://dx.doi.org/10.1164/rccm.200910-15000C

39. Hanania NA, Donohue JF, Nelson H, et al. The safety and efficacy of arformoterol and formoterol in COPD. COPD. 2010; 7(1): 17-31. doi: http://dx.doi.org/10.3109/15412550903499498

40. Anzueto A, Ferguson GT, Feldman G, et al. Effect of fluticasone propionate/salmeterol (250/50) on COPD exacerbations and impact on patient outcomes. COPD. 2009; 6(5): 320-329. doi: http://dx.doi.org/10.1080/15412550903140881

41. Rennard SI, Tashkin DP, McElhattan J, et al. Efficacy and tolerability of budesonide/formoterol in one hydrofluoroalkane pressurized metered-dose inhaler in patients with chronic obstructive pulmonary disease: results from a 1-year randomized controlled clinical trial. Drugs. 2009; 69(5): 549-565. doi: http://dx.doi.org/10.2165/00003495-200969050-00004.

42. Donohue JF, Hanania NA, Sciarappa KA, et al. Arformoterol and salmeterol in the treatment of chronic obstructive pulmonary disease: a one year evaluation of safety and tolerance. Ther Adv Respir Dis. 2008; 2(2): 37-48. doi: http://dx.doi.org/10.1177/1753465808089455.

43. Ferguson GT, Anzueto A, Fei R, et al. Effect of fluticasone propionate/salmeterol (250/50 microg) or salmeterol (50 microg) on COPD exacerbations. Respir Med. 2008; 102(8): 10991108. doi: http://dx.doi.org/10.1016/j.rmed.2008.04.019

44. Tashkin DP, Rennard SI, Martin P, et al. Efficacy and safety of budesonide and formoterol in one pressurized metereddose inhaler in patients with moderate to very severe chronic obstructive pulmonary disease: results of a 6-month randomized clinical trial. Drugs. 2008; 68(14): 1975-2000. doi: http://dx.doi.org/10.2165/00003495-200868140-00004

45. Tashkin DP, Celli B, Senn S, et al. A 4-year trial of tiotropium in chronic obstructive pulmonary disease. N Engl J Med. 2008; 359(15): 1543-1554.

doi: http://dx.doi.org/10.1056/NEJMoa0805800 
46. Tonnel AB, Perez T, Grosbois JM, et al. Effect of tiotropium on health-related quality of life as a primary efficacy endpoint in COPD. Int J Chron Obstruct Pulmon Dis. 2008; 3(2): 301-310. doi: http://dx.doi.org/10.2147/COPD.S2463

47. Aaron SD, Vandemheen KL, Fergusson D, et al. Tiotropium in combination with placebo, salmeterol, or fluticasone-salmeterol for treatment of chronic obstructive pulmonary disease: a randomized trial. Ann Intern Med. 2007; 146(8): 545-555. doi: http://dx.doi.org/10.7326/0003-4819-146-8-200704170-00152

48. Chan CK, Maltais F, Sigouin C, Haddon JM, Ford GT, on behalf of the SAFE Study Group. A randomized controlled trial to assess the efficacy of tiotropium in Canadian patients with chronic obstructive pulmonary disease. Can Respir J. 2007; 14(8): 465-472.

49. Stockley RA, Chopra N, Rice L. Addition of salmeterol to existing treatment in patients with COPD: a 12 month study. Thorax. 2006; 61(2): 122-128.

doi: http://dx.doi.org/10.1136/thx.2004.033266

50. GlaxoSmithKline. A multicentre, randomised, doubleblind, parallel group, 24-week study to compare the effect of the salmeterol/fluticasone propionate combination product $50 / 250 \mathrm{mcg}$, with salmeterol $50 \mathrm{mcg}$ both delivered twice daily via the DISKUS/ACCUHALER inhaler on lung function and dyspnoea in subjects with chronic obstructive pulmonary disease (COPD). GlaxoSmithKline Clinical Trial Register website. http://www.gsk-clinicalstudyregister.com/ study/100470\#rs2005 Published 2005. Accessed February 25, 2016.

51. GlaxoSmithKline. Multicentre, randomised, parallel group, placebo-controlled, double-blind study, stratified on tobacco status at enrollment, evaluating during 6 months the efficacy of salmeterol powder for inhalation, 50ug two times per day for the reduction of thoracic distension in subjects with chronic obstructive pulmonary disease (COPD). GlaxoSmithKline Clinical Trial Register website. http://www. gsk-clinicalstudyregister.com/study/SLMF4010\#rs Published 2005. Accessed February 25, 2016.

52. Wouters EF, Postma DS, Fokkens B, et al. Withdrawal of fluticasone propionate from combined salmeterol/fluticasone treatment in patients with COPD causes immediate and sustained disease deterioration: a randomised controlled trial. Thorax. 2005; 60(6): 480-487.

doi: http://dx.doi.org/10.1136/thx.2004.034280

53. Brusasco V, Hodder R, Miravitlles M, et al. Health outcomes following treatment for six months with once daily tiotropium compared with twice daily salmeterol in patients with COPD. Thorax. 2003; 58(5): 399-404.

doi: http://dx.doi.org/10.1136/thx.58.5.399

54. Calverley P, Pauwels R, Vestbo J, et al. Combined salmeterol and fluticasone in the treatment of chronic obstructive pulmonary disease: a randomised controlled trial. Lancet. 2003; 361(9356): 449-456.

doi: http://dx.doi.org/10.1016/S0140-6736(03)12459-12462
55. Dal Negro RW, Pomari C, Tognella S, Micheletto C. Salmeterol \& fluticasone $50 \mathrm{microg} / 250 \mathrm{microg}$ bid in combination provides a better long-term control than salmeterol 50 microg bid alone and placebo in COPD patients already treated with theophylline. Pulm Pharmacol Ther. 2003; 16(4): 241-246.

56. Hanania NA, Darken P, Horstman D, et al. The efficacy and safety of fluticasone propionate (250 microg)/salmeterol (50 microg) combined in the Diskus inhaler for the treatment of COPD. Chest. 2003; 124(3): 834-843. doi: http://dx.doi.org/10.1378/chest.124.3.834

57. Casaburi R, Mahler DA, Jones PW, et al. A long-term evaluation of once-daily inhaled tiotropium in chronic obstructive pulmonary disease. Eur Respir J. 2002; 19(2): 217-224. doi: http://dx.doi.org/10.1183/09031936.02.00269802

58. Chapman KR, Arvidsson P, Chuchalin AG, et al. The addition of salmeterol 50 microg bid to anticholinergic treatment in patients with COPD: a randomized, placebo controlled trial. Chronic obstructive pulmonary disease. Can Respir J. 2002; 9(3): 178-185.

59. Donohue JF, van Noord JA, Bateman ED, et al. A 6-month, placebo-controlled study comparing lung function and health status changes in COPD patients treated with tiotropium or salmeterol. Chest. 2002; 122(1): 47-55.

doi: http://dx.doi.org/10.1378/chest.122.1.47

60. Mahler DA, Wire P, Horstman D, et al. Effectiveness of fluticasone propionate and salmeterol combination delivered via the Diskus device in the treatment of chronic obstructive pulmonary disease. Am J Respir Crit Care Med. 2002; 166(8): 1084-1091.

doi: http://dx.doi.org/10.1164/rccm.2112055

61. Rossi A, Kristufek P, Levine BE, et al. Comparison of the efficacy, tolerability, and safety of formoterol dry powder and oral, slowrelease theophylline in the treatment of COPD. Chest. 2002; 121(4): 1058-1069.

doi: http://dx.doi.org/10.1378/chest.121.4.1058

62. Vincken W, van Noord JA, Greefhorst AP, et al. Improved health outcomes in patients with COPD during 1 yr's treatment with tiotropium. Eur Respir J. 2002; 19(2): 209-216. doi: http://dx.doi.org/10.1183/09031936.02.00238702

63. Spencer S, Calverley PM, Sherwood Burge P, Jones PW. Health status deterioration in patients with chronic obstructive pulmonary disease. Am J Respir Crit Care Med. 2001; 163(1): 122-128. doi: http://dx.doi.org/10.1164/ajrccm.163.1.2005009

64. Ferrer M, Alonso J, Prieto L, et al. Validity and reliability of the St George's Respiratory Questionnaire after adaptation to a different language and culture: the Spanish example. Eur Respir J. 1996; 9(6): 1160-1166. doi: http://dx.doi.org/10.1183/09031936.96.09061160 
65. Stahl E, Lindberg A, Jansson SA, et al. Health-related quality of life is related to COPD disease severity. Health Qual Life Outcomes. 2005; 3:56. doi: http://dx.doi.org/10.1186/1477-7525-3-56

66. Eltayara L, Becklake MR, Volta CA, Milic-Emili J. Relationship between chronic dyspnea and expiratory flow limitation in patients with chronic obstructive pulmonary disease. Am J Respir Crit Care Med. 1996; 154(6): 1726-1734. doi: http://dx.doi.org/10.1164/ajrccm.154.6.8970362

67. Westwood M, Bourbeau J, Jones PW, et al. Relationship between $\mathrm{FEV}_{1}$ change and patient-reported outcomes in randomised trials of inhaled bronchodilators for stable COPD: a systematic review. Respiratory Res. 2011; 12:40. doi: http://dx.doi.org/10.1186/1465-9921-12-40

68. Berthelot JM, Le Goff B, Maugars Y. The Hawthorne effect: stronger than the placebo effect? Joint Bone Spine. 2011; 78(4): 335-336.

doi: http://dx.doi.org/10.1016/j.jbspin.2011.06.001

69. Maleki-Yazdi MR, Kaelin T, Richard N, Zvarich M, Church A. Efficacy and safety of umeclidinium/vilanterol $62.5 / 25 \mathrm{mcg}$ and tiotropium $18 \mathrm{mcg}$ in chronic obstructive pulmonary disease: results of a 24-week, randomized, controlled trial. Respir Med. 2014; 108(12): 1752-1760.

doi: http://dx.doi.org/10.1016/j.rmed.2014.10.002 